\title{
Growth optimal investment in discrete-time markets with proportional transaction costs
}

\author{
N. Denizcan Vanli ${ }^{a, *}$, Sait Tunc ${ }^{b}$, Mehmet A. Donmez ${ }^{c}$, Suleyman S. Kozat ${ }^{a}$ \\ a Department of Electrical and Electronics Engineering, Bilkent University, Ankara, Turkey \\ ${ }^{\mathrm{b}}$ Department of Industrial and Systems Engineering, University of Wisconsin-Madison, WI, USA \\ ${ }^{c}$ Department of Electrical and Computer Engineering, University of Illinois at Urbana-Champaign, IL, USA
}

\section{A R T I C L E I N F O}

\section{Article history:}

Available online 19 October 2015

\section{Keywords:}

Growth optimal portfolio

Threshold rebalancing

Proportional transaction cost

Discrete-time stock market

\begin{abstract}
A B S T R A C T
We investigate how and when to diversify capital over assets, i.e., the portfolio selection problem, from a signal processing perspective. To this end, we first construct portfolios that achieve the optimal expected growth in i.i.d. discrete-time two-asset markets under proportional transaction costs. We then extend our analysis to cover markets having more than two stocks. The market is modeled by a sequence of price relative vectors with arbitrary discrete distributions, which can also be used to approximate a wide class of continuous distributions. To achieve the optimal growth, we use threshold portfolios, where we introduce a recursive update to calculate the expected wealth. We then demonstrate that under the threshold rebalancing framework, the achievable set of portfolios elegantly form an irreducible Markov chain under mild technical conditions. We evaluate the corresponding stationary distribution of this Markov chain, which provides a natural and efficient method to calculate the cumulative expected wealth. Subsequently, the corresponding parameters are optimized yielding the growth optimal portfolio under proportional transaction costs in i.i.d. discrete-time two-asset markets. As a widely known financial problem, we also solve the optimal portfolio selection problem in discrete-time markets constructed by sampling continuous-time Brownian markets. For the case that the underlying discrete distributions of the price relative vectors are unknown, we provide a maximum likelihood estimator that is also incorporated in the optimization framework in our simulations.
\end{abstract}

(C) 2015 Elsevier Inc. All rights reserved.

\section{Introduction}

The problem of how and when an investor should diversify capital over various assets, whose future returns are yet to be realized, is extensively studied in various different fields from signal processing $[1,2,12,28-30,33,35]$ and financial engineering [25, $26]$ to machine learning [13,34] and information theory [9]. Naturally, this is one of the most important financial applications due to the amount of money involved. However, the recent financial crisis demonstrated that there is a significant room for improvement in this field by sound signal processing methods [12,30], which is the main goal of this paper. In this paper, we investigate how and when to diversify capital over assets, i.e., the portfolio selection problem, from a signal processing perspective and provide portfolio selection strategies that maximize the expected cumula-

\footnotetext{
* Corresponding author.

E-mail addresses: vanli@ee.bilkent.edu.tr (N.D. Vanli), stunc@wisc.edu (S. Tunc), donmez2@illinois.edu (M.A. Donmez), kozat@ee.bilkent.edu.tr (S.S. Kozat).
}

tive wealth in discrete-time markets under proportional transaction costs.

In particular, we study an investment problem in markets that allows trading at discrete periods, where the discrete period is arbitrary, e.g., it can be seconds, minutes or days [24]. Furthermore, the market levies transaction fees for both selling and buying an asset proportional to the volume of trading at each transaction, which accurately models a broad range of financial markets [3,24]. In our discussions, we first consider markets with two assets. Twostock markets are extensively studied in financial literature and are shown to accurately model a wide range of financial applications [24] such as the well-known "Stock and Bond Market" [24]. We then extend our analysis to markets having more than two assets, i.e., $m$-stock markets, where $m$ is arbitrary.

Following the extensive literature [9,19,24-26,33], the market is modeled by a sequence of price relative vectors, say $\{\boldsymbol{X}(n)\}_{n \geq 1}$, $\boldsymbol{X}(n) \in[0, \infty)^{m}$, where each entry of $\boldsymbol{X}(n)$, i.e., $X_{i}(n) \in[0, \infty)$, is the ratio of the closing price to the opening price of the $i$ th stock per investment period. Hence, each entry of $\boldsymbol{X}(n)$ quantifies the gain (or the loss) of that asset at each investment period. The sequence of price relative vectors is assumed to have an i.i.d. "dis- 
crete" distribution [24-26,33], however, the discrete distributions on the vector of price relatives are arbitrary. In this sense, the corresponding discrete distributions can approximate a wide class of continuous distributions on the price relatives that satisfy certain regularity conditions by appropriately increasing the size of the discrete sample space. We first assume that the discrete distributions on the price relative vectors are known and then extend our analysis to cover the case, where the underlying distributions are unknown. We emphasize that the i.i.d. assumption on the sequence of price relative vectors is shown to hold in most realistic markets [14,24].

At each investment period, the diversification of the capital over the assets is represented by a portfolio vector $\boldsymbol{b}(n)$, where $\forall i \in\{1, \ldots, m\}, b_{i}(n)$ represents the ratio of the capital invested in the $i$ th asset at investment period $n$, i.e., we have $\sum_{i=1}^{m} b_{i}(n)=1$, where $0 \leq b_{i}(n) \leq 1$. As an example, if we invest using $\boldsymbol{b}(n)$, we earn (or lose) $\boldsymbol{b}^{T}(n) \boldsymbol{X}(n)$ at the $n$th investment period, after $\boldsymbol{X}(n)$ is revealed. Given that we start with one dollar, after an investment period of $N$ days, we have the wealth growth $\prod_{n=1}^{N} \boldsymbol{b}^{T}(n) \boldsymbol{X}(n)$. Under this general market model, we provide algorithms that maximize the expected growth over any period $N$ by using "threshold rebalanced portfolios" (TRPs), which are shown to yield optimal growth in general i.i.d. discrete-time markets [14].

In [9], Cover et al. showed that the portfolio that achieves the maximal growth is a constant rebalanced portfolio (CRP) in i.i.d. discrete-time markets, under certain assumptions on the sequence of price relatives and without any transaction costs. A CRP is a portfolio investment strategy, where the fraction of wealth invested in each stock is kept constant at each investment period. A problem extensively studied in this framework is to find sequential portfolios that asymptotically achieve the wealth of the best CRP tuned to the underlying sequence of price relatives. Several sequential algorithms are introduced to achieve the performance of the best CRP (such as $[9,13,16,34]$ ) with different convergence rates and different performances on historical data sets. In [3], sequential algorithms that achieve the performance of the best CRP under transaction costs are introduced. However, we emphasize that keeping a CRP may require extensive trading due to a possible rebalancing at each investment period which deems CRPs (even the best CRP) ineffective in realistic markets even under mild transaction costs [19].

In continuous-time markets, however, it has been shown that under transaction costs, the optimal portfolios that achieve the maximal wealth are certain class of "no-trade zone" portfolios $[7,11,32]$. In simple terms, a no-trade zone portfolio has a compact closed set and a rebalancing occurs if the current portfolio breaches this set, otherwise no rebalancing occurs. Clearly, such a no-trade zone portfolio may avoid hefty transaction costs since it can limit excessive rebalancing by defining appropriate no-trade zones. Analogous to continuous time markets, it has been shown in [14] that in two-asset i.i.d. markets under proportional transaction costs, compact no-trade zone portfolios are optimal such that they achieve the maximal growth under certain assumptions on the sequence of price relatives. In two-asset markets, the compact no-trade zone is represented by thresholds, e.g., if at investment period $n$, the portfolio is given by $\boldsymbol{b}(n)=[b(n),(1-b(n))]^{T}$, where $0 \leq b(n) \leq 1$, then rebalancing occurs if $b(n) \notin(\alpha, \beta)$, given the thresholds $\alpha, \beta$, where $0 \leq \alpha \leq \beta \leq 1$. Similarly, the interval $(\alpha, \beta)$ can be represented using a target portfolio $b$ and a region around it, i.e., $(b-\epsilon, b+\epsilon)$, where $0 \leq \epsilon \leq \min \{b, 1-b\}$ such that $\alpha=b-\epsilon$ and $\beta=b+\epsilon$. Extension of TRPs to markets having more than two stocks is straightforward and explained in Section 3.2.

However, how to construct the no-trade zone portfolio, i.e., how to select the thresholds that achieve the maximal growth, has not yet been solved except in elementary scenarios [14]. In [15], a universal algorithm that asymptotically achieves the performance of the best TRP tuned to the underlying sequence of price relatives is introduced. This algorithm leverages Bayesian type weighting from [9] inspired from universal source coding and requires no statistical assumptions on the sequence of price relatives. In similar lines, various different universal algorithms are introduced that achieve the performance of the best expert in different competition classes in $[1,2,17-20]$. Although the performance guarantees in $[1,2,15,18,19])$ are derived without any stochastic assumptions, these methods are highly conservative due to the worst case scenario optimization, i.e., they are only optimal in an asymptotical sense. However, the order of such performance upper bounds may not be negligible in actual financial markets [6,20], even though they may be neglected in source coding applications (where these algorithms are inspired from). We demonstrate that our algorithm readily outperforms these universal methods over historical data.

Our main contributions are as follows. We first consider twoasset markets and recursively evaluate the expected achieved wealth of a threshold portfolio for any $b$ and $\epsilon$ over any investment period. We then extend this analysis to markets having more than two-stocks. We next demonstrate that under the threshold rebalancing framework, the achievable set of portfolios form an irreducible Markov chain under mild technical conditions. We evaluate the corresponding stationary distribution of this Markov chain, which provides a natural and efficient method to calculate the cumulative expected wealth. Subsequently, the corresponding parameters are optimized using a brute force approach yielding the growth optimal investment portfolio under proportional transaction costs in i.i.d. discrete-time two-asset markets. We note that for the case with the irreducible Markov chain, which covers practically all scenarios in the realistic markets, the optimization of the parameters is offline and carried out only once. However, for the case with recursive calculations, the algorithm has an exponential computational complexity in terms of the number of states. However, in our simulations, we observe that a reduced complexity form of the recursive algorithm that keeps only a constant number of states by appropriately pruning certain states provides nearly identical results with the "optimal" algorithm. Furthermore, as a well studied problem, we also solve optimal portfolio selection in discrete-time markets constructed by sampling continuous-time Brownian markets [24]. When the underlying discrete distributions of the price relative vectors are unknown, we provide a maximum likelihood estimator to estimate the corresponding distributions that is incorporated in the optimization framework in the Simulations section. For all these approaches, we also provide the corresponding complexity bounds.

The organization of the paper is as follows. In Section 2, we briefly describe our discrete-time stock market model with discrete price relatives and symmetric proportional transaction costs. In Section 3, we start to investigate TRPs, where we first introduce a recursive update in Section 3.1 for a market having twostocks. Generalization of the iterative algorithm to the $m$-asset market case is provided in Section 3.2. We then show that the TRP framework can be analyzed using finite state Markov chains in Section 3.4 and Section 3.5. The special Brownian market is analyzed in Section 3.6. The maximum likelihood estimator is derived in Section 4. We simulate the performance of our algorithms in Section 5 and conclude the paper with certain remarks in Section 6 .

\section{Problem description}

We consider discrete-time stock markets under transaction costs. We first consider a market with two stocks and then extend the analysis to markets having more than two stocks. We model the market using a sequence of price relative vectors $\mathbf{X}(n)$. 
A vector of price relatives $\mathbf{X}(n)=\left[X_{1}(n), \ldots, X_{m}(n)\right]^{T}$ in a market of $m$ assets represents the change in the prices of the assets over investment period $n$, i.e., for the $i$ th stock $X_{i}(n)$ is the ratio of the closing to the opening price of the $i$ th stock over period $n$. For a market having two assets, we have $\mathbf{X}(n)=\left[X_{1}(n) X_{2}(n)\right]^{T}$. We assume that the price relative sequences $X_{1}(n)$ and $X_{2}(n)$ are i.i.d. over with possibly different discrete sample spaces $\mathcal{X}_{1}$ and $\mathcal{X}_{2}$, i.e., $X_{1}(n) \in \mathcal{X}_{1}$ and $X_{2}(n) \in \mathcal{X}_{2}$, respectively [14]. For technical reasons, in our derivations, we assume that the sample space is $\mathcal{X} \triangleq \mathcal{X}_{1} \cup \mathcal{X}_{2}=\left\{x_{1}, x_{2}, \ldots, x_{K}\right\}$ for both $X_{1}(n)$ and $X_{2}(n)$ where $|\mathcal{X}|=K$ is the cardinality of the set $\mathcal{X}$. The probability mass function (pmf) of $X_{1}(n)$ is $p_{1}(x) \triangleq \operatorname{Pr}\left(X_{1}=x\right)$ and the pmf of $X_{2}(n)$ is $p_{2}(x) \triangleq \operatorname{Pr}\left(X_{2}=x\right)$. We define $p_{i, 1}=p_{1}\left(x_{i}\right)$ and $p_{i, 2}=p_{2}\left(x_{i}\right)$ for $x_{i} \in \mathcal{X}$ and the probability mass vectors $\mathbf{p}_{1}=\left[\begin{array}{llll}p_{1,1} & p_{2,1} \ldots & p_{K, 1}\end{array}\right]^{T}$ and $\mathbf{p}_{2}=\left[\begin{array}{llll}p_{1,2} & p_{2,2} \ldots & p_{K, 2}\end{array}\right]^{T}$, respectively. Here, we first assume that the corresponding probability mass vectors $\mathbf{p}_{1}$ and $\mathbf{p}_{2}$ are known. We then extend our analysis to the case, where $\mathbf{p}_{1}$ and $\mathbf{p}_{2}$ are unknown, and sequentially estimate $\mathbf{p}_{1}$ and $\mathbf{p}_{2}$ using a maximum likelihood estimator in Section 4.

An allocation of wealth over two stocks is represented by the portfolio vector $\mathbf{b}(n)=\left[b_{1}(n), b_{2}(n)\right]^{T}$, where $b_{1}(n)$ and $b_{2}(n)$ represents the proportion of wealth invested in the first and second stocks, respectively, at investment period $n$. In two stock markets, we have $b_{2}(n)=1-b_{1}(n)$, thus $\mathbf{b}(n)$ is completely characterized by the proportion of the total wealth invested in the first stock, i.e., $b_{1}(n)$. For notational clarity, we use $b(n)$ instead of $b_{1}(n)$ throughout the paper, hence our portfolio vector is denoted by $\mathbf{b}(n)=[b(n), 1-b(n)]^{T}$.

We denote a threshold rebalancing portfolio with an initial and target portfolio $b$ and a threshold $\epsilon$ by $\operatorname{TRP}(b, \epsilon)$. At each market period $n$, an investor rebalances the asset allocation only if the portfolio leaves the interval $(b-\epsilon, b+\epsilon)$. When $b(n) \notin$ ( $b-\epsilon, b+\epsilon)$, the investor buys and sells stocks so that the asset allocation is rebalanced to the initial allocation, i.e., $b(n)=b$, where the investor has to pay transaction fees. We emphasize that the rebalancing can be made directly to the closest boundary instead of to $b$ as suggested in [14], however, we rebalance to $b$ for notational simplicity, whereas our derivations also hold for the rebalancing to the boundary case. We model the transaction cost of rebalancing by a fixed proportional cost $c \in(0,1)[3,14,19]$. As an example, if the investor buys or sells $S$ dollars of stocks, then he pays $c S$ dollars of transaction fees. Although we assume a symmetric transaction cost ratio, all the results can be carried over to markets with asymmetric costs $[14,19]$.

We let $S(N)$ denote the achieved wealth at investment period $N$ and assume, without loss of generality, that the initial wealth of the investor is 1 dollar. Then, as an example, if the portfolio $b(n)$ does not leave the interval $(b-\epsilon, b+\epsilon)$ and the allocation of wealth is not rebalanced for $N$ investment periods, then the achieved wealth is given by $S(N)=b \prod_{n=1}^{N} X_{1}(n)+$ $(1-b) \prod_{n=1}^{N} X_{2}(n)$ and the current proportion of wealth invested in the first stock is given by $b(N)=\left[b \prod_{n=1}^{N} X_{1}(n)\right] / S(N)$. On the other hand, if the portfolio leaves the interval $(b-\epsilon, b+\epsilon)$ at period $N$, i.e., $b(N) \notin(b-\epsilon, b+\epsilon)$, then the investor rebalances the asset distribution to the initial distribution and pays $c S(N)|b(N)-b|$ dollars for transaction costs [3]. In the next section, we first derive a compact form for the expected achieved wealth $E[S(N)]$ so that we can optimize $b$ and $\epsilon$ to maximize $E[S(N)]$.

\section{Threshold rebalanced portfolios}

In this section, we first investigate TRPs in discrete-time twoasset markets under proportional transaction costs. We first introduce an iterative method to calculate the expected achieved wealth

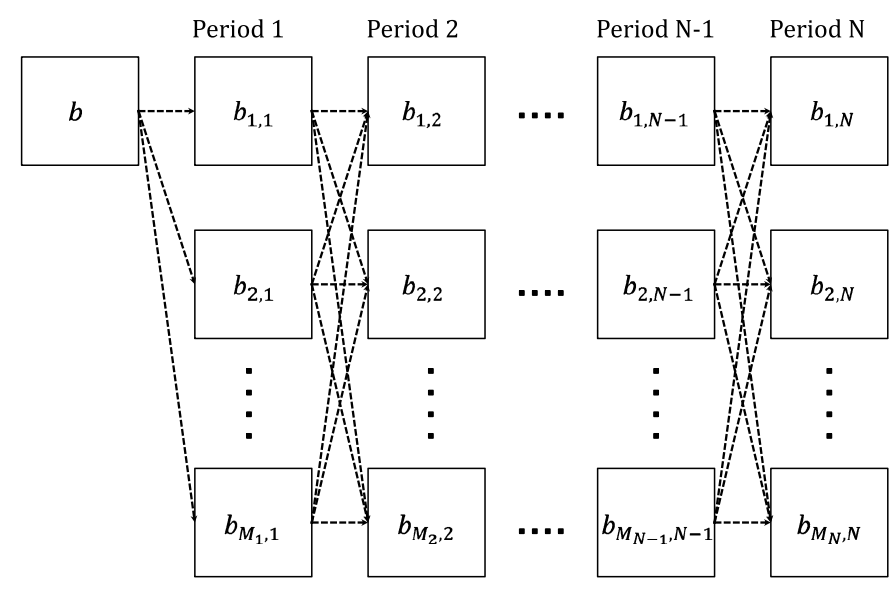

Fig. 1. Block diagram representation of $N$ period investment.

at a given investment period. We then present an upper bound on the complexity of this algorithm. We next calculate the expected achieved wealth of markets having more than two assets, i.e., $m$-asset markets for an arbitrary $m$. We then provide the necessary and sufficient conditions such that the achievable portfolios are finite such that the complexity of the algorithm does not grow at any period. We also show that the portfolio sequence converges to a stationary distribution and derive the expected achieved wealth. Based on the calculation of the expected achieved wealth, we optimize $b$ and $\epsilon$ using a brute-force search. Finally, with these derivations, we consider the well-known discrete-time two-asset Brownian market with proportional transaction costs and investigate the asymptotic expected achieved wealth.

\subsection{An iterative algorithm}

Before introducing the iterative algorithm, we first define the set of achievable portfolios at each investment period since the iterative calculation of the expected achieved wealth is based on the achievable portfolio set. We then introduce the portfolio transition sets and the transition probabilities of achievable portfolios at successive investment periods in order to iteratively find the probability of each portfolio state and to calculate $E[S(N)]$.

We define the set of achievable portfolios at each investment period as follows. As $|\mathcal{X}|=K<\infty$, the set of achievable portfolios at period $N$ can only have finitely many elements. We define the set of achievable portfolios at period $N$ as $\mathcal{B}_{N}=\left\{b_{1, N}, \ldots, b_{M_{N}, N}\right\}$, where $M_{N} \triangleq\left|\mathcal{B}_{N}\right|$. As illustrated in Fig. 1, for each achievable portfolio $b_{m, N} \in \mathcal{B}_{N}, m=1, \ldots, M_{N}$, there is a certain set of portfolios in $\mathcal{B}_{N-1}$ that are connected to $b_{m, n}$. At a given investment pe$\operatorname{riod} N$, the set of achievable portfolios $\mathcal{B}_{N}$ is given by

$$
\begin{gathered}
\mathcal{B}_{N}=\{b\} \cup\left\{b_{m, N}: b_{m, N}=\frac{b_{k, N-1} u}{b_{k, N-1} u+\left(1-b_{k, N-1}\right) v}\right. \\
\in(b-\epsilon, b+\epsilon), u, v \in \mathcal{X}\} .
\end{gathered}
$$

Since $b$ is an achievable portfolio at each $N$ (due to a possible rebalancing), without loss of generality, we let $b_{1, N}=b$ for each $N \in \mathbb{N}$. Note that in Fig. 1 , the size of the set of achievable portfolios may grow as $N$ increases.

Having constructed a state transition diagram, we next represent the transition probabilities from $b_{k, N-1}$ to $b_{m, N}$ as follows

$q_{k, m, N} \triangleq \operatorname{Pr}\left(b(N)=b_{m, N} \mid b(N-1)=b_{k, N-1}\right)$,

where $k=1, \ldots, M_{N-1}$ and $m=1, \ldots, M_{N}$. Given that $b(N)=$ $b_{m, N}$, for some $m=1, \ldots, M_{N}$, there exists a corresponding set of portfolios $\mathcal{N}_{m, N} \subset \mathcal{B}_{N-1}$ from which $b_{m, N}$ is achievable, i.e., 
$\mathcal{N}_{m, N} \triangleq\left\{b_{k, N-1} \in \mathcal{B}_{N-1}: q_{k, m, N}>0, k=1, \ldots, M_{N-1}\right\}$

where $m=1, \ldots, M_{N}$. Then, the probability of each portfolio state can be calculated as follows

$\operatorname{Pr}\left(b(N)=b_{m, N}\right)=\sum_{b_{k, N-1} \in \mathcal{N}_{m, N}} q_{k, m, N} \operatorname{Pr}\left(b(N-1)=b_{k, N-1}\right)$

where $m=1, \ldots, M_{N}$. By the definition of $\mathcal{B}_{N}$ and using the law of total expectation [31], we can write

$E[S(N)]=\sum_{m=1}^{M_{N}} \operatorname{Pr}\left(b(N)=b_{m, N}\right) E\left[S(N) \mid b(N)=b_{m, N}\right]$.

To obtain an iterative formulation using (5), we next find the transition probabilities between achievable portfolios.

To accomplish this, we let $\mathcal{U}_{k, m, N}$ denote the set of price relative vectors that connect $b_{k, N-1}$ to $b_{m, N}$, i.e.,

$\mathcal{U}_{k, m, N} \triangleq\left\{\mathbf{w}=\left[w_{1}, w_{2}\right]^{T} \in \mathcal{X}^{2}:\right.$

$$
\left.\frac{w_{1} b_{k, N-1}}{w_{1} b_{k, N-1}+w_{2}\left(1-b_{k, N-1}\right)}=b_{m, N}\right\}
$$

for $k=1, \ldots, M_{N-1}$ and $m=2, \ldots, M_{N}$. We consider the price relative vectors that connect $b_{k, N-1}$ to $b_{1, N}=b$ separately since, in this case, there are two subcases depending on whether the portfolio leaves the interval $(b-\epsilon, b+\epsilon)$ or not. We let $\mathcal{U}_{k, 1, N}=\mathcal{V}_{k, 1, N} \cup \mathcal{R}_{k, 1, N}$, where $\mathcal{V}_{k, 1, N}$ is the set of price relative vectors that connect $b_{k, N-1}$ to $b_{1, N}=b$ without rebalancing, i.e.,

$\mathcal{V}_{k, 1, N}=\left\{\mathbf{w}=\left[w_{1}, w_{2}\right]^{T} \in \mathcal{X}^{2}: \frac{w_{1} b_{k, N-1}}{w_{1} b_{k, N-1}+w_{2}\left(1-b_{k, N-1}\right)}=b\right\}$,

and $\mathcal{R}_{k, 1, N}$ is the set of price relative vectors that connect $b_{k, N-1}$ to $b_{1, N}$ with rebalancing, i.e.,

$$
\begin{aligned}
\mathcal{R}_{k, 1, N}= & \left\{\mathbf{w}=\left[w_{1}, w_{2}\right]^{T} \in \mathcal{X}^{2}:\right. \\
& \left.\frac{w_{1} b_{k, N-1}}{w_{1} b_{k, N-1}+w_{2}\left(1-b_{k, N-1}\right)} \notin(b-\epsilon, b+\epsilon)\right\} .
\end{aligned}
$$

With these definitions, we can obtain the transition probabilities as follows

$$
\begin{aligned}
q_{k, m, N} & =\operatorname{Pr}\left(\mathbf{X}(N) \in \mathcal{U}_{k, m, N}\right) \\
& =\sum_{\mathbf{w} \in \mathcal{U}_{k, m, N}} p_{1}\left(w_{1}\right) p_{2}\left(w_{2}\right),
\end{aligned}
$$

where $k=1, \ldots, M_{N-1}$ and $m=1, \ldots, M_{N}$.

Having derived a recursive formulation for the state probabilities, we can calculate the term in the sum in (5) by considering two separate cases as follows.

i) As the first case, we consider $b(N)=b_{m, N}$, where $m=$ $2, \ldots, M_{N}$, i.e., we know that the portfolio does not leave the interval $(b-\epsilon, b+\epsilon)$ at period $N$. Therefore, no transaction cost is paid and we have

$$
\begin{aligned}
& \operatorname{Pr}\left(b(N)=b_{m, N}\right) E\left[S(N) \mid b(N)=b_{m, N}\right] \\
& =\sum_{b_{k, N-1} \in \mathcal{N}_{m, N}} E\left[S(N) \mid b(N)=b_{m, N}, b(N-1)=b_{k, N-1}\right] \\
& \quad \times \operatorname{Pr}\left(b(N)=b_{m, N}\right) \operatorname{Pr}\left(b(N-1)=b_{k, N-1} \mid b(N)=b_{m, N}\right)
\end{aligned}
$$

$$
\begin{aligned}
& =\sum_{b_{k, N-1} \in \mathcal{N}_{m, N}} E\left[S(N) \mid b(N)=b_{m, N}, b(N-1)=b_{k, N-1}\right] \\
& \quad \times \operatorname{Pr}\left(b(N-1)=b_{k, N-1}\right) q_{k, m, N}, \\
& =\sum_{b_{k, N-1} \in \mathcal{N}_{m, N}} E\left[S(N) \mid b(N)=b_{m, N}, b(N-1)=b_{k, N-1}\right] \\
& \quad \times \operatorname{Pr}\left(b(N-1)=b_{k, N-1}\right) \operatorname{Pr}\left(\mathbf{X}(N) \in \mathcal{U}_{k, m, N}\right) \\
& =\sum_{b_{k, N-1} \in \mathcal{N}_{m, N}} \sum_{\mathbf{w} \in \mathcal{U}_{k, m, N}} E\left[S(N) \mid b(N)=b_{m, N}, b(N-1)=b_{k, N-1},\right. \\
& \quad \times \operatorname{Pr}\left(b(N-1)=b_{k, N-1}\right) \operatorname{Pr}\left(\mathbf{X}(N)=\mathbf{w} \mid \mathbf{X}(N) \in \mathcal{U}_{k, m, N}\right) \\
& \quad \times \operatorname{Pr}\left(\mathbf{X}(N) \in \mathcal{U}_{k, m, N}\right),
\end{aligned}
$$

where (9) and (12) follow from the law of total expectation [31], (10) follows from Bayes' theorem [31], and (11) follows from (2). As no rebalancing occurs, we also have

$$
\begin{gathered}
E\left[S_{N} \mid b(N)=b_{m, N}, b(N-1)=b_{k, N-1}, \mathbf{X}(N)=\mathbf{w}\right] \\
=E\left[S(N-1)\left(b_{k, N-1} w_{1}+\left(1-b_{k, N-1}\right) w_{2}\right) \mid\right. \\
\left.b(N-1)=b_{k, N-1}\right] .
\end{gathered}
$$

Putting (13) back in (12), we obtain

$$
\begin{aligned}
\operatorname{Pr} & \left(b(N)=b_{m, N}\right) E\left[S(N) \mid b(N)=b_{m, N}\right] \\
= & \sum_{b_{k, N-1} \in \mathcal{N}_{m, N}} \sum_{\mathbf{w} \in \mathcal{U}_{k, m, N}} \operatorname{Pr}\left(b(N-1)=b_{k, N-1}\right) \operatorname{Pr}(\mathbf{X}(N)=\mathbf{w}) \\
& \times E\left[S(N-1)\left(b_{k, N-1} w_{1}+\left(1-b_{k, N-1}\right) w_{2}\right) \mid\right. \\
& \left.\quad b(N-1)=b_{k, N-1}\right] \\
= & \sum_{b_{k, N-1} \in \mathcal{N}_{m, N}} \operatorname{Pr}\left(b(N-1)=b_{k, N-1}\right) \\
& \times E\left[S(N-1) \mid b(N-1)=b_{k, N-1}\right] \\
& \times \sum_{\mathbf{w} \in \mathcal{U}_{k, m, N}}\left(b_{k, N-1} w_{1}+\left(1-b_{k, N-1}\right) w_{2}\right) p_{1}\left(w_{1}\right) p_{2}\left(w_{2}\right)
\end{aligned}
$$

where the last line follows since $\operatorname{Pr}(\mathbf{X}(N)=\mathbf{w})=p_{1}\left(w_{1}\right) p_{2}\left(w_{2}\right)$.

ii) In the second case, we have $b(N)=b_{1, N}$. For this case, if $\mathbf{X}(N) \in \mathcal{V}_{k, 1, N}$, then the portfolio is not rebalanced and no transaction fee is paid; whereas if $\mathbf{X}(N) \in \mathcal{R}_{k, 1, N}$, then the portfolio is rebalanced and transaction cost is paid. Thus, we have

$$
\begin{aligned}
& \operatorname{Pr}\left(b(N)=b_{1, N}\right) E\left[S(N) \mid b(N)=b_{1, N}\right] \\
& =\sum_{b_{k, N-1} \in \mathcal{N}_{1, N}} E\left[S(N) \mid b(N)=b_{1, N}, b(N-1)=b_{k, N-1}\right] \\
& \quad \times \operatorname{Pr}\left(b(N-1)=b_{k, N-1}\right) q_{k, 1, N} \\
& =\sum_{b_{k, N-1} \in \mathcal{N}_{1, N}}\left\{\sum_{\mathbf{w} \in \mathcal{V}_{k, 1, N}} \operatorname{Pr}\left(b(N-1)=b_{k, N-1}\right) \operatorname{Pr}(\mathbf{X}(N)=\mathbf{w})\right. \\
& \quad \times E\left[S_{N} \mid b(N)=b_{1, N}, b(N-1)=b_{k, N-1}, \mathbf{X}(N)=\mathbf{w}\right] \\
& \quad+\sum_{\mathbf{w} \in \mathcal{R}_{k, 1, N}} \operatorname{Pr}\left(b(N-1)=b_{k, N-1}\right) \operatorname{Pr}(\mathbf{X}(N)=\mathbf{w}) \\
& \left.\quad \times E\left[S_{N} \mid b(N)=b_{1, N}, b(N-1)=b_{k, N-1}, \mathbf{X}(N)=\mathbf{w}\right]\right\}
\end{aligned}
$$


where (16) follows from (10) and the last line follows in similar lines to (14). Here, when $\mathbf{X}(N)=\mathbf{w} \in \mathcal{V}_{k, 1, N}$, no rebalancing occurs. Thus, we have

$$
\begin{gathered}
E\left[S_{N} \mid b(N)=b_{1, N}, b(N-1)=b_{k, N-1}, \mathbf{X}(N)=\mathbf{w}\right] \\
=E\left[S(N-1)\left(b_{k, N-1} w_{1}+\left(1-b_{k, N-1}\right) w_{2}\right) \mid\right. \\
\left.b(N-1)=b_{k, N-1}\right] .
\end{gathered}
$$

On the other hand, when $\mathbf{X}(N)=\mathbf{w} \in \mathcal{R}_{k, 1, N}$, rebalancing occurs. Hence, we have

$$
\begin{aligned}
& E\left[S_{N} \mid b(N)=b_{1, N}, b(N-1)=b_{k, N-1}, \mathbf{X}(N)=\mathbf{w}\right] \\
& =E\left[S(N-1)\left(b_{k, N-1} w_{1}+\left(1-b_{k, N-1}\right) w_{2}\right)\right. \\
& \quad \times\left(1-c\left|\frac{b_{k, N-1} w_{1}}{b_{k, N-1} w_{1}+\left(1-b_{k, N-1}\right) w_{2}}-b\right|\right) \mid \\
& \left.b(N-1)=b_{k, N-1}\right] .
\end{aligned}
$$

Putting (18) and (19) back in (17), we obtain

$$
\begin{aligned}
& \operatorname{Pr}\left(b(N)=b_{1, N}\right) E\left[S(N) \mid b(N)=b_{1, N}\right] \\
& =\sum_{b_{k, N-1} \in \mathcal{N}_{1, N}} \operatorname{Pr}\left(b(N-1)=b_{k, N-1}\right)\left\{\sum_{\mathbf{w} \in \mathcal{V}_{k, 1, N}} \operatorname{Pr}(\mathbf{X}(N)=\mathbf{w})\right. \\
& \times E\left[S(N-1)\left(b_{k, N-1} w_{1}+\left(1-b_{k, N-1}\right) w_{2}\right) \mid\right. \\
& \left.\quad b(N-1)=b_{k, N-1}\right] \\
& +\sum_{\mathbf{w} \in \mathcal{R}_{k, 1, N}} \operatorname{Pr}(\mathbf{X}(N)=\mathbf{w}) \\
& \times E\left[S(N-1)\left(b_{k, N-1} w_{1}+\left(1-b_{k, N-1}\right)\right)\right. \\
& \times\left(1-c\left|\frac{b_{k, N-1} w_{1}}{b_{k, N-1} w_{1}+\left(1-b_{k, N-1}\right) w_{2}}-b\right|\right) \mid \\
& \left.\left.\quad b(N-1)=b_{k, N-1}\right]\right\} .
\end{aligned}
$$

After some algebra, we obtain

$$
\begin{aligned}
\operatorname{Pr}( & \left.b(N)=b_{1, N}\right) E\left[S(N) \mid b(N)=b_{1, N}\right] \\
= & \sum_{b_{k, N-1} \in \mathcal{N}_{1, N}} \operatorname{Pr}\left(b(N-1)=b_{k, N-1}\right) \\
& \times E\left[S(N-1) \mid b(N-1)=b_{k, N-1}\right] \\
& \times\left\{\sum_{\mathbf{w} \in \mathcal{V}_{k, 1, N}}\left(b_{k, N-1} w_{1}+\left(1-b_{k, N-1}\right) w_{2}\right) p_{1}\left(w_{1}\right) p_{2}\left(w_{2}\right)\right. \\
& +\sum_{\mathbf{w} \in \mathcal{R}_{k, 1, N}}\left(b_{k, N-1} w_{1}+\left(1-b_{k, N-1}\right) w_{2}\right) p_{1}\left(w_{1}\right) p_{2}\left(w_{2}\right) \\
& \left.\times\left(1-c\left|\frac{b_{k, N-1} w_{1}}{b_{k, N-1} w_{1}+\left(1-b_{k, N-1}\right) w_{2}}-b\right|\right)\right\} .
\end{aligned}
$$

Hence, we can iteratively calculate (5) $i$ ) using (15), for the case where $b(N)=b_{l, N}$ and $l=2, \ldots M_{N}$, ii) using (20), for the case where $b(N)=b_{1, N}=b$. Since we have the recursive formulation, we can optimize $b$ and $\epsilon$ by a brute force search as shown in the Simulations section. In the following section, we extend our analyses to the $m$-asset market case.

\subsection{Generalization of the iterative algorithm to the $m$-asset market case}

In this section, we generalize our iterative method introduced in Section 3.1 for a market with $m$ assets where $m \in \mathbb{Z}^{+}$. We model the market as a sequence of i.i.d. price relative vectors $\mathbf{X}(n)=\left[X_{1}(n), \ldots, X_{m}(n)\right]$, where $X_{i}(n) \in \mathcal{X}$ and the pmf of $X_{i}(n)$ is $p_{i}(x)=\operatorname{Pr}\left(X_{i}(n)=x\right)$. For the $m$-asset case, the portfolio vector is given by $\mathbf{b}(n)=\left[b_{1}(n), \ldots, b_{m}(n)\right]$, where $\sum_{i=1}^{m} b_{i}(n)=1$ and $b_{i}(n) \geq 0$, the target portfolio vector is defined as $\mathbf{b}=\left[b_{1}, \ldots, b_{m}\right]$ and the threshold vector is given by $\epsilon=\left[\epsilon_{1}, \ldots, \epsilon_{m}\right]$. Similar to the two-asset case, $\operatorname{TRP}(\mathbf{b}, \boldsymbol{\epsilon})$ rebalances the wealth allocation $\mathbf{b}(n)$ to b only when $\mathbf{b}(n) \notin \mathbf{b}^{\epsilon} \triangleq\left(b_{1}-\epsilon_{1}, b_{1}+\epsilon_{1}\right) \times\left(b_{2}-\epsilon_{2}, b_{2}+\epsilon_{2}\right) \times$ $\ldots \times\left(b_{m}-\epsilon_{m}, b_{m}+\epsilon_{m}\right)$. In this case, if the wealth allocation is not rebalanced for $N$ investment periods, then the achieved wealth is given by $S(N)=\sum_{k=1}^{m} b_{k} \prod_{n=1}^{N} X_{k}(N)$ and the proportion of wealth invested in the $i$ th asset becomes $b_{i}(N)=\frac{b_{i} \prod_{n=1}^{N} X_{i}(N)}{\sum_{k=1}^{m} b_{k} \prod_{n=1}^{N} X_{k}(N)}$. We define the set of achievable portfolios at period $N$ as

$\mathcal{B}_{N}=\{\mathbf{b}\} \cup\left\{\mathbf{b}_{m, N}: \mathbf{b}_{k, N}=\frac{\mathbf{b}_{m, N-1} \circ \mathbf{x}}{\mathbf{x}^{T} \mathbf{b}_{m, N-1}} \in \mathbf{b}^{\epsilon}, \mathbf{x} \in \mathcal{X}^{m}\right\}$,

where $\mathbf{b} \circ \mathbf{x} \triangleq\left[b_{1} x_{1}, \ldots, b_{m} x_{m}\right]^{T}$ is an elementwise multiplication operation. In accordance with the definitions given in two-asset market case, the definitions of the portfolio transition sets and the transition probabilities of achievable portfolios follow. Then, similar to the iterative algorithm introduced in Section 3.1, i.e., (15) and (20), we can iteratively calculate the following expected achieved wealth

$E[S(N)]=\sum_{l=1}^{M_{N}} \operatorname{Pr}\left(\mathbf{b}(N)=\mathbf{b}_{l, N}\right) E\left[S(N) \mid \mathbf{b}(N)=\mathbf{b}_{l, N}\right]$.

We emphasize that the complete iterations for the $m$-asset case can be obtained by changing the scalars in (15) and (20) by the corresponding vectors. For the rest of the paper, we consider twoasset markets for ease of exposition.

\subsection{Complexity analysis of the iterative algorithm}

In this section, we investigate the number of achievable portfolios at a given market period to determine the complexity of the iterative algorithm. We show that the set of achievable portfolios at period $N$ is equivalent to the set of achievable portfolios when the portfolio $b(n)$ does not leave the interval $(b-\epsilon, b+\epsilon)$ for $N$ investment periods. We first demonstrate that if the portfolio never leaves the interval $(b-\epsilon, b+\epsilon)$ for $N$ periods, then $b(N)$ is given by $b(N)=1 /\left(1+\frac{1-b}{b} e^{\sum_{n=1}^{N} Z(n)}\right)$, where $Z(n) \triangleq \ln \frac{X_{2}(n)}{X_{1}(n)}$ with a sample space $\mathcal{Z} \triangleq\left\{z=\ln \frac{u}{v} \mid u, v \in \mathcal{X}\right\}$ and $|\mathcal{Z}|=M$. Then, we argue that the number of achievable portfolios at period $N$, i.e., $M_{N}$, is equal to the number of different values that the sum $\sum_{n=1}^{N} Z(n)$ can take when the portfolio does not leave the interval $(b-\epsilon, b+\epsilon)$ for $N$ investment periods. We then observe that we have $M \leq K^{2}-K+1$ as the price relative sequences $X_{1}(n)$ and $X_{2}(n)$ are elements of the same sample space $\mathcal{X}$ with $|\mathcal{X}|=K$. Using this inequality, we finally find an upper bound on the number of achievable portfolios.

Lemma 1. The number of achievable portfolios at period $N$, i.e., $M_{N}$, is equal to the number of different values that the sum $\sum_{n=1}^{N} Z(n)$ can take when the portfolio $b(n)$ does not leave the interval $(b-\epsilon, b+\epsilon)$ for $N$ investment periods and is bounded by $\left(\begin{array}{c}N+K^{2}-K \\ N\end{array}\right)$, i.e., $M_{N}=\left|\mathcal{B}_{N}\right| \leq$ $\left(\begin{array}{c}N+K^{2}-K \\ N\end{array}\right)$.

Proof. The proof is in Appendix A. 
Remark 1. Note that the complexity of calculating $E[S(N)]$ is bounded by $\mathcal{O}\left(\sum_{n=1}^{N}\left(\begin{array}{c}n+K^{2}-K \\ n\end{array}\right) / N\right)$ since at each period $n=$ $1, \ldots, N$, we calculate $E[S(n)]$ as a summation of $M_{n}$ terms in (5) and $M_{n} \leq\left(\begin{array}{c}n+K^{2}-K \\ n\end{array}\right)$.

As can be observed from Remark 1, the number of achievable portfolios tends to go to the infinity as $N$ increases. However, in the next section, we show that the number of achievable portfolios is finite under mild technical conditions, hence the computational complexity of our algorithm is constant.

\subsection{Finitely many achievable portfolios}

In this section, we investigate the cardinality of the set of achievable portfolios $\mathcal{B} \triangleq \bigcup_{n=1}^{\infty} \mathcal{B}_{n}$ and demonstrate that $\mathcal{B}$ is finite under certain conditions. Consequently, when $\mathcal{B}$ is finite, we can derive a recursive update with a constant complexity to calculate the expected achieved wealth $E[S(n)]$ at any investment period. To show this, we demonstrate that the portfolio sequence forms a Markov chain with a finite state space and converges to a stationary distribution. Then, we investigate the limiting behavior of the expected achieved wealth using this update to optimize $b$ and $\epsilon$. Before providing the main theorem, we first state a couple of lemmas that are used in the derivation of the main result of this section.

We first show that the portfolio $b(n)$ does not leave the interval $(b-\epsilon, b+\epsilon)$ for $N$ periods iff the sum $\sum_{n=1}^{k} Z(n) \in$ $\left(\alpha^{-}, \alpha^{+}\right)$for $k=1, \ldots, N$, where $\alpha^{-} \triangleq \ln \frac{b(1-b-\epsilon)}{(1-b)(b+\epsilon)}<0$ and $\alpha^{+} \triangleq$ $\ln \frac{b(1-b+\epsilon)}{(1-b)(b-\epsilon)}>0$. We then prove that the number of achievable portfolios is equal to the cardinality of the set $\mathcal{Y} \cap\left(\alpha^{-}, \alpha^{+}\right)$, where

$\mathcal{Y} \triangleq\left\{\sum_{i=1}^{M^{+}} y_{i} z_{i}: y_{i} \in \mathbb{Z}, z_{i} \in \mathcal{Z}^{+}, i=1, \ldots, M^{+}\right\}$,

$\mathcal{Z}^{+} \triangleq\{z \in \mathcal{Z}: z \geq 0\}$, and $M^{+} \triangleq\left|\mathcal{Z}^{+}\right|$. Note that $\mathcal{Z}^{+}$is the set of positive elements of the set $\mathcal{Z}$ and any value that the sum $\sum_{n=1}^{N} Z(n)$ can take is an element of $\mathcal{Y}$. Hence, if we can demonstrate that the set $\mathcal{Y} \cap\left(\alpha^{-}, \alpha^{+}\right)$is finite under certain conditions, then it yields that the cardinality of the set $\mathcal{B}$ is also finite.

Lemma 2. The portfolio $b(n)$ does not leave the interval $(b-\epsilon, b+\epsilon)$ for $N$ investment periods iff the sum $\sum_{n=1}^{k} Z(n) \in\left(\alpha^{-}, \alpha^{+}\right)$, where $k=$ $1, \ldots, N$.

Proof. The proof is in Appendix B.

In the following lemma, we demonstrate that the set of different values that $\sum_{n=1}^{N} Z(n)$ can take for any $N \in \mathbb{N}$, when the portfolio never leaves the interval $(b-\epsilon, b+\epsilon)$ for $N$ investment periods is equivalent to the set $\mathcal{Y} \cap\left(\alpha^{-}, \alpha^{+}\right)$, when $|z|<\min \left\{\left|\alpha^{-}\right|,\left|\alpha^{+}\right|\right\}$. Hence, we show that the cardinality of the set of achievable portfolios is equal to the cardinality of the set $\mathcal{Y} \cap\left(\alpha^{-}, \alpha^{+}\right)$.

Lemma 3. If $|z|<\min \left\{\left|\alpha^{-}\right|,\left|\alpha^{+}\right|\right\}, \forall z \in \mathcal{Z}^{+}$, then $\forall y \in \mathcal{Y} \cap\left(\alpha^{-}, \alpha^{+}\right)$, $\exists\left\{Z(n)=Z^{(n)}\right\}_{n=1}^{N} \in \mathcal{Z}$ for some $N \in \mathbb{N}$ such that $y=\sum_{n=1}^{N} Z^{(n)}$ and $\sum_{n=1}^{k} Z^{(n)} \in\left(\alpha^{-}, \alpha^{+}\right), \forall k=1, \ldots, N$.

Proof. The proof is in Appendix C.

This lemma illustrates that the set of different values that the sum $\sum_{n=1}^{N} Z(n)$ can take for any $N \in \mathbb{N}$ when the portfolio does not leave the interval $(b-\epsilon, b+\epsilon)$ for $N$ investment periods is equivalent to the set $\mathcal{Y} \cap\left(\alpha^{-}, \alpha^{+}\right)$. Thus, the number of achievable portfolios is equal to the cardinality of the set $\mathcal{Y} \cap\left(\alpha^{-}, \alpha^{+}\right)$. In the following theorem, we demonstrate that if $|z|<\min \left\{\left|\alpha^{-}\right|,\left|\alpha^{+}\right|\right\}$, $\forall z \in \mathcal{Z}^{+}$, and the set $\mathcal{Y}$ has a minimum positive element, then $\mathcal{Y} \cap\left(\alpha^{-}, \alpha^{+}\right)$is finite. Hence, the set of achievable portfolios is also finite under these conditions. Otherwise, we show that the set $\mathcal{Y} \cap\left(\alpha^{-}, \alpha^{+}\right)$contains countably infinite elements.

Theorem 1. If $|z|<\min \left\{\left|\alpha^{-}\right|,\left|\alpha^{+}\right|\right\}, \forall z \in \mathcal{Z}^{+}$, and the set $\mathcal{Y}$ has a minimum positive element, i.e., $\exists \delta=\min \{y \in \mathcal{Y}: y>0\}$, then the set of achievable portfolios $\mathcal{B}=\cup_{n=1}^{\infty} \mathcal{B}_{n}$ is finite. If such a minimum positive element does not exist, then $\mathcal{B}$ is countably infinite.

Proof. The proof is in Appendix D.

In Theorem 1 we present a sufficient and necessary condition for the achievable portfolios to be finite. We emphasize that the required condition, i.e., $|z|<\min \left\{\left|\alpha^{-}\right|,\left|\alpha^{+}\right|\right\}, \forall z \in \mathcal{Z}^{+}$, is a necessary required technical condition which assures that the TRP thresholds are large enough to prohibit constant rebalancings at each investment period. In this sense, this condition does not limit the generality of the TRP framework.

By Theorem 1, we establish the conditions for a unique stationary distribution of the achievable portfolios. With the existence of a unique stationary distribution, in the next section, we provide the asymptotic behavior of the expected wealth growth by presenting the growth rate. However, before proceeding further, we note that although Theorem 1 states that the number of achievable portfolios is finite under certain conditions, it does not specify the exact number. In the following corollary, we demonstrate that the number of achievable portfolios is $\left\lfloor\frac{\alpha^{+}-\alpha^{-}}{\delta}\right\rfloor$ if the set of achievable portfolios is finite.

Corollary 1. If $|z|<\min \left\{\left|\alpha^{-}\right|,\left|\alpha^{+}\right|\right\}, \forall z \in \mathcal{Z}^{+}$, and $\exists \delta=\min \{y \in \mathcal{Y}$ : $y>0\}$, then the number of achievable portfolios is $\left\lfloor\frac{\alpha^{+}-\alpha^{-}}{\delta}\right\rfloor{ }^{1}$

Proof. The proof is in Appendix E.

In Theorem 1, we introduce conditions on the cardinality of the set of all achievable portfolio states, $\mathcal{B}$, and showed that $\mathcal{B}$ is finite under certain conditions. Using this result, we next analyze the asymptotic behavior of the expected achieved wealth, i.e., we demonstrate that when $\mathcal{B}$ is finite, the portfolio sequence converges to a stationary distribution. Hence, we can determine the limiting behavior of the expected achieved wealth so that we can optimize $b$ and $\epsilon$. To accomplish this, we first present a recursive update to evaluate $E[S(n)]$. We then maximize

$g(b, \epsilon) \triangleq \lim _{n \rightarrow \infty} \frac{1}{n} \log E[S(n)]$

over $b$ and $\epsilon$ with a brute-force search, i.e., we calculate $g(b, \epsilon)$ for different $(b, \epsilon)$ pairs and find the one that yields the maximum expected wealth.

\subsection{Finite state Markov chain for threshold portfolios}

When $\mathcal{B}$ is finite, it follows from Corollary 1 that there are exactly $L \triangleq\left\lfloor\frac{\alpha^{+}-\alpha^{-}}{\delta}\right\rfloor$ achievable portfolios. Then, we let $\mathcal{B}=$ $\left\{b_{1}, \ldots, b_{L}\right\}$, where $b_{1}=b$ without loss of generality. We define the probability mass vector of the portfolio sequence as $\boldsymbol{\pi}(n)=$

\footnotetext{
1 Here, $\lfloor x / y\rfloor$ is the largest integer less than or equal to $x / y$ and $\lceil x / y\rceil$ is the smallest integer greater or equal to the $x / y$.
} 
$\left[\pi_{1}(n), \ldots, \pi_{L}(n)\right]^{T}$ where $\pi_{i}(n) \triangleq \operatorname{Pr}\left(b(n)=b_{i}\right)$. The portfolio sequence $b(n)$ forms a homogeneous Markov chain with a finite state space $\mathcal{B}$ since the transition probabilities between states are independent of period $n$. We observe that $b(n)$ is irreducible since each state communicates with other states so that all states are nullpersistent as $\mathcal{B}$ is finite [31]. Then, it follows that there exists a unique stationary distribution vector $\pi=\lim _{n \rightarrow \infty} \pi(n)$. To calculate $\pi$, we first observe that the set of portfolios from which $b_{m}$ is achievable, i.e., $\mathcal{N}_{m, n}$, and the set of price relative vectors that connect $b_{k}$ to $b_{m}$, i.e., $\mathcal{U}_{k, m, n}$, are independent of the investment period $n$ since the price relative sequences are i.i.d. $\forall k, m \in\{1, \ldots, L\}$. Hence, we drop the subscript $n$ from these terms for ease of exposition and write $\mathcal{U}_{k, m, n}=\mathcal{U}_{k, m}$ and $\mathcal{N}_{m, n}=\mathcal{N}_{m}, \forall n \in \mathbb{N}$. We next note that the state transition probabilities are also independent of $n$, hence we also have $q_{k, m, n}=q_{k, m}, \forall n \in \mathbb{N}$ and $\forall n \in \mathbb{N}$. Therefore, we can write $\operatorname{Pr}\left(b(n)=b_{m}\right)$ as follows

$\operatorname{Pr}\left(b(n)=b_{m}\right)=\sum_{k=1}^{L} q_{k, m} \operatorname{Pr}\left(b(n-1)=b_{k}\right)$,

where $q_{k, m}=0$ if $b_{k} \notin \mathcal{N}_{m}$. Now, by using the definition of $\pi(n)$ and (25), we get $\boldsymbol{\pi}(n+1)=\mathbf{P} \boldsymbol{\pi}(n), \forall n \geq 0$, where $\mathbf{P}$ is the state transition matrix, i.e., $\mathbf{P}_{i j}=q_{i, j}$.

In order to determine the limiting behavior of $E[S(n)]$, we first define the vector $\mathbf{e}(n)=\left[e_{1}(n), \ldots, e_{L}(n)\right]^{T}$, where $e_{i}(n) \triangleq$ $\operatorname{Pr}\left(b(n)=b_{i}\right) \times E\left[S(n) \mid b(n)=b_{i}\right]$. Then, from (5), we have $E[S(n)]=$ $\sum_{i=1}^{L} e_{i}(n)=\mathbf{1}^{T} \mathbf{e}(n)$, where $\mathbf{1}$ is the vector of ones. Hence, according to the definition of $\mathbf{e}(n)$ and using (15) and (20), we can write $\mathbf{e}(n+1)=\mathbf{Q} \mathbf{e}(n)$, where

$\mathbf{Q} \triangleq\left[\begin{array}{ccc}\sum_{\mathbf{w} \in \mathcal{U}_{1,1}} \kappa_{1} & \cdots & \sum_{\mathbf{w} \in \mathcal{U}_{L, 1}} \kappa_{L} \\ \vdots & \ddots & \vdots \\ \sum_{\mathbf{w} \in \mathcal{U}_{1, L}} \kappa_{1} & \cdots & \sum_{\mathbf{w} \in \mathcal{U}_{L, L}} \kappa_{L}\end{array}\right]$,

and $\kappa_{i} \triangleq\left(b_{i} w_{1}+\left(1-b_{i}\right) w_{2}\right) p_{1}\left(w_{1}\right) p_{2}\left(w_{2}\right)$, where we ignore rebalancing for presentation purposes. If we take rebalancing into account, then only the first row of $\mathbf{Q}$ changes as follows

$$
\begin{aligned}
{[\mathbf{Q}]_{1, m}=} & \sum_{\mathbf{w} \in \mathcal{V}_{m, 1}}\left(b_{1} w_{1}+\left(1-b_{1}\right) w_{2}\right) p_{1}\left(w_{1}\right) p_{2}\left(w_{2}\right) \\
& +\sum_{\mathbf{w} \in \mathcal{R}_{m, 1}}\left(b_{1} w_{1}+\left(1-b_{1}\right) w_{2}\right) \\
& \times\left(1-c\left|\frac{b_{1} w_{1}}{b_{1} w_{1}+\left(1-b_{1}\right) w_{2}}-b\right|\right) p_{1}\left(w_{1}\right) p_{2}\left(w_{2}\right),
\end{aligned}
$$

where $\mathcal{V}_{m, 1}$ is the set of price relative vectors that connect $b_{m}$ to $b_{1}=b$ without crossing the threshold boundaries and $\mathcal{R}_{m, 1}$ is the set of price relative vectors that connect $b_{m}$ to $b_{1}=b$ by crossing the threshold boundaries. ${ }^{2}$

Here, we analyze $E[S(n)]$ as $n \rightarrow \infty$ as follows. We assume that the matrix $\mathbf{Q}$ is diagonalizable with eigenvalues $\lambda_{1}, \ldots, \lambda_{L}$, where, without loss of generality, we assume that $\lambda_{L} \leq \cdots \leq \lambda_{1}$, which is the case for a wide range of transaction costs [31]. Then, there exists a nonsingular matrix B such that $\mathbf{Q}=\mathbf{B} \boldsymbol{\Lambda} \mathbf{B}^{-1}$ where $\boldsymbol{\Lambda}$ is the diagonal matrix with entries $\lambda_{1}, \ldots, \lambda_{L}$. We observe that $\mathbf{Q}$ has nonnegative entries. Therefore, it follows from Perron-Frobenius Theorem [27] that $\mathbf{Q}$ has a unique largest eigenvalue $\lambda_{1}>0$ and any other eigenvalue is strictly smaller than $\lambda_{1}$ in absolute value, i.e., $\lambda_{1}>\left|\lambda_{j}\right|, \forall j \in\{2, \ldots, L\}$. Then, the recursion on $\boldsymbol{e}(n)$ yields $\mathbf{e}(n)=\mathbf{Q}^{n} \mathbf{e}(0)$, and $E[S(n)]$ can be written as follows

${ }^{2}$ Here, $[\mathbf{Q}]_{i, j}$ represents the entry of the matrix $\mathbf{Q}$ at its $i$ th row and $j$ th column.
$E[S(n)]=\mathbf{1}^{T} \mathbf{Q}^{n} \mathbf{e}(0)=\mathbf{1}^{T} \mathbf{B} \mathbf{\Lambda}^{n} \mathbf{B}^{-1} \mathbf{e}(0)=\sum_{i=1}^{L} u_{i} v_{i} \lambda_{i}^{n}$,

where $\mathbf{u} \triangleq\left[u_{1}, \ldots, u_{L}\right]^{T}=\mathbf{B}^{T} \mathbf{1}$ and $\mathbf{v} \triangleq\left[v_{1}, \ldots, v_{L}\right]=\mathbf{B}^{-1} \mathbf{e}(0)$. Then, it follows that

$$
\begin{aligned}
g(b, \epsilon) & =\lim _{n \rightarrow \infty} \frac{1}{n} \log \left\{\sum_{i=1}^{L} u_{i} v_{i} \lambda_{i}^{n}\right\} \\
& =\lim _{n \rightarrow \infty} \log \lambda_{1}+\lim _{n \rightarrow \infty} \frac{1}{n} \log \left\{\sum_{i=1}^{L} u_{i} v_{i}\left(\frac{\lambda_{i}}{\lambda_{1}}\right)^{n}\right\} \\
& =\log \lambda_{1},
\end{aligned}
$$

since $\lim _{n \rightarrow \infty}\left(\frac{\lambda_{i}}{\lambda_{1}}\right)^{n}=0, \forall i \in\{2, \ldots, L\}$. Hence, we can optimize $b$ and $\epsilon$ as follows

$$
\begin{aligned}
{\left[b^{*}, \epsilon^{*}\right] } & =\underset{b \in[0,1], 0<\epsilon}{\arg \max } g(b, \epsilon) \\
& =\underset{b \in[0,1], 0<\epsilon}{\arg \max } \log \lambda_{1} .
\end{aligned}
$$

To maximize $g(b, \epsilon)$, we evaluate it for different values of $(b, \epsilon)$ pairs and find the pair that maximizes $g(b, \epsilon)$, i.e., by a brute-force search in the Simulations section.

In the next section, we investigate the well-studied two-asset Brownian market model under transaction costs.

\subsection{Two stock Brownian markets}

In this section, we consider the well-known two-asset Brownian market, where the stock price signals are generated from a standard Brownian motion [11,14,32]. Portfolio selection problem in continuous time two-asset Brownian markets with proportional transaction costs was investigated in [32], where the growth optimal investment strategy is shown to be a threshold portfolio. Here, as usually done in the financial literature [11], we first convert the continuous time Brownian market by sampling to a discretetime market [14]. We then calculate the expected achieved wealth and optimize $b$ and $\epsilon$ to find the best portfolio rebalancing strategy for a discrete-time Brownian market with transaction costs. We emphasize that although, the growth optimal investment in discrete-time two-asset Brownian markets with proportional transaction costs was investigated in [14], the expected achieved wealth and the optimal threshold interval $(b-\epsilon, b+\epsilon)$ has not been calculated yet.

To model the Brownian two-asset market, we use the price relative vector $\mathbf{X}=\left[X_{1}, X_{2}\right]^{T}$, with $X_{1}=1$ and $X_{2}=e^{a Z}$ where $a$ is a constant and $Z$ is a random variable with $\operatorname{Pr}(Z= \pm 1)=\frac{1}{2}$. This price relative vector is obtained by sampling the stock price processes of the continuous time two-asset Brownian market [14, 32]. We emphasize that this sampling results a discrete-time market identical to the binomial model popular in asset pricing [14]. Under this framework, we next present the set of achievable portfolios and the transition probabilities between portfolio states.

Since the price of the first stock is the same over investment periods, the portfolio leaves the interval $(b-\epsilon, b+\epsilon)$ if either the money in the second stock grows over a certain limit or falls below a certain limit. If the portfolio $b(n)$ does not leave the interval $(b-\epsilon, b+\epsilon)$ for $N$ investment periods, then the money in the first stock is $b$ dollars and the money in the second stock is $(1-b) e^{a k}$ dollars for some $-N \leq k \leq N$ so that the portfolio is $b(N)=\frac{b}{b+(1-b) e^{a k}}$. Note that $b(N) \in(b-\epsilon, b+\epsilon)$ iff $k_{\min } \leq k \leq$ $k_{\max }$, where $k_{\min } \triangleq\left\lceil\frac{1}{a} \ln \frac{b(1-b-\epsilon)}{(1-b)(b+\epsilon)}\right]$ and $k_{\max } \triangleq\left\lfloor\frac{1}{a} \ln \frac{b(1-b+\epsilon)}{(1-b)(b-\epsilon)}\right\rfloor$. Hence, the set of achievable portfolios is given by 
$\mathcal{B}=\left\{b_{k}=\frac{b}{b+(1-b) e^{a\left(k+k_{\min }-1\right)}}: k=1, \ldots, k_{\max }-k_{\min }+1\right\}$.

For ease of exposition, we denote $\mathcal{B}=\left\{b_{1}, \ldots, b_{L}\right\}$, where $L=$ $|\mathcal{B}|=k_{\max }-k_{\min }+1$ and $b_{1-k_{\min }}=b$, without loss of generality. We see that the portfolio is rebalanced to $b_{1-k_{\min }}=b$ only if it is in the state $b_{1}$ and $X_{2}=e^{-a}$ or it is in the state $b_{L}$ and $X_{2}=e^{a}$. Therefore, the transition probabilities are given by

$q_{k, m}= \begin{cases}\frac{1}{2}, & \text { if }|k-m|=1 \text { or } k \in\{1, L\} \text { and } m=1-k_{\min } \\ 0, & \text { otherwise, }\end{cases}$

where $q_{k, m}$ is the probability that we have $b(n)=b_{m}$ given that $b(n-1)=b_{k}$ at any period $n$. We then calculate $E[S(n)]$ using the recursions in Section 3.5 as follows. The sets of price relative vectors that connect portfolio states are given by

$\mathcal{U}_{k, m}= \begin{cases}{\left[1, e^{a}\right]^{T},} & \text { if } k \in\{1, \ldots, L-1\}, m=k+1 \text { or } k=L, \\ {\left[1, e^{-a}\right]^{T},} & \text { if } k \in\{2, \ldots, L\}, m=k-1 \text { or } k=1, \\ & m=1-k_{\min } \\ \emptyset, & \text { otherwise. }\end{cases}$

Hence, we can calculate the matrix $\mathbf{Q}$ defined in (26) as follows

$[\mathbf{Q}]_{m, k}= \begin{cases}\frac{1}{2}\left(b_{k}+\left(1-b_{k}\right) e^{a}\right), & \text { if } m \in\{2, \ldots, L\} \text { and } k=m-1 \\ \frac{1}{2}\left(b_{k}+\left(1-b_{k}\right) e^{-a}\right), & \text { if } m \in\{1, \ldots, L-1\} \text { and } \\ 0, & k=m+1 \\ 0, & \text { otherwise, }\end{cases}$

where we ignore rebalancing. If we take rebalancing into account, then we can write

$[\mathbf{Q}]_{1-i_{\min }, 1}=\frac{1}{2}\left(b_{1}+\left(1-b_{1}\right) e^{-a}\right)\left(1-c\left|\frac{b_{1}}{b_{1}+\left(1-b_{1}\right) e^{-a}}-b\right|\right)$,

and

$[\mathbf{Q}]_{1-i_{\min }, L}=\frac{1}{2}\left(b_{S}+\left(1-b_{L}\right) e^{a}\right)\left(1-c\left|\frac{b_{L}}{b_{L}+\left(1-b_{L}\right) e^{a}}-b\right|\right)$.

Then, using the recursions in Section 3.5, we can obtain $E[S(n)]=$ $\mathbf{Q}^{n} \mathbf{e}(0)$. Then, we maximize $g(b, \epsilon)=\lim _{n \rightarrow \infty} \frac{1}{n} \log E[S(n)]=$ $\log \lambda_{1}$, where $\lambda_{1}$ is the largest eigenvalue of $\mathbf{Q}$. Here, we optimize $b$ and $\epsilon$ with a brute-force search, i.e., we find $\lambda_{1}$ for different $(b, \epsilon)$ pairs and find the one that achieves the maximum.

\section{Maximum likelihood estimation of the probability mass vectors}

In this section, we sequentially estimate the probability mass vectors $\mathbf{p}_{1}$ and $\mathbf{p}_{2}$ corresponding to $X_{1}(n)$ and $X_{2}(n)$, respectively, using a maximum likelihood estimator (MLE). In general, these vectors may not be known or may change in time, hence, they should be estimated at each investment period prior to the calculation of $E[S(n)]$. The MLE for a pmf on a finite set is wellknown [31], but we provide the corresponding derivations here for completeness. We consider the price relative sequence $\left\{X_{1}(n)\right\}_{n=1}^{N}$, where $X_{1}(n)=w_{n} \in \mathcal{X}$, for $n=1, \ldots, N$ is an arbitrary realization of this sequence. Here, our aim is to estimate $\mathbf{p}_{1}$, where we point out that similar derivations follow for the price relative sequence $X_{2}(n)$ and $\mathbf{p}_{2}$.

As demonstrated in our simulations, the corresponding estimation can be carried out over a finite length window to emphasize the most recent data. We let $\mathbf{w} \triangleq\left[w_{1}, \ldots, w_{N}\right]^{T}$ represent the realization vector and $\boldsymbol{\theta} \triangleq\left[\theta_{x_{1}}, \ldots, \theta_{x_{K}}\right]^{T}$ represent the parameter vector, where $\theta_{x_{i}} \triangleq p_{1}\left(x_{i} \mid \boldsymbol{\theta}\right)$ is the conditional pmf for $i=1, \ldots, K$. Then, the MLE of the $\mathbf{p}_{1}$ is given by

$$
\begin{aligned}
\hat{\boldsymbol{\theta}}_{\mathrm{ML}}=\underset{\sum_{i=1}^{K} \theta_{x_{i}}=1: \theta_{x_{i}} \geq 0}{\arg \max } p_{1}(\mathbf{w} \mid \boldsymbol{\theta}) \\
=\underset{\sum_{i=1}^{K} \theta_{x_{i}}=1: \theta_{x_{i}} \geq 0}{\arg \max } \operatorname{Pr}\left(X_{1}(1)=w_{1}, \ldots, X_{1}(N)=w_{N} \mid \boldsymbol{\theta}\right) .
\end{aligned}
$$

Since the price relative sequence $X_{1}(n)$ is i.i.d., it follows that $p_{1}(\mathbf{w} \mid \boldsymbol{\theta})=\prod_{i=1}^{N} p_{1}\left(w_{i} \mid \boldsymbol{\theta}\right)=\prod_{i=1}^{N} \theta_{w_{i}}=\prod_{i=1}^{N} \prod_{j=1}^{K} \theta_{x_{j}}^{\mathrm{I}\left(w_{i}=x_{j}\right)}$, where $\mathrm{I}(\cdot)$ is the indicator function. If we change the order of the product operators, then we obtain $p_{1}(\mathbf{w} \mid \boldsymbol{\theta})=\prod_{i=1}^{N} \prod_{j=1}^{K} \theta_{x_{j}}^{\mathrm{I}\left(w_{i}=x_{j}\right)}=$ $\prod_{j=1}^{K} \theta_{x_{j}}^{\sum_{i=1}^{N} \mathrm{I}\left(w_{i}=x_{j}\right)}=\prod_{j=1}^{K} \theta_{x_{j}}^{N_{j}}$, where $N_{j} \triangleq \sum_{i=1}^{N} \mathrm{I}\left(w_{i}=x_{j}\right)$ represents the number of realizations that are equal to $x_{j} \in \mathcal{X}$ for $j=1, \ldots, K$. Noting that $\sum_{j=1}^{K} N_{j}=N$, we can write $\hat{\boldsymbol{\theta}}_{\mathrm{ML}}=$ $\arg \max _{\theta} \sum_{j=1}^{K} \frac{N_{j}}{N} \log \theta_{x_{j}}$, since $\log (\cdot)$ is a monotone increasing function. If we define the vector $\mathbf{h} \triangleq\left[h_{x_{1}}, \ldots, h_{x_{K}}\right]^{T}$, where $h_{x_{j}} \triangleq \frac{N_{j}}{N}$ for $j=1, \ldots, K$, then we observe that $h_{x_{j}} \geq 0$ for $j=1, \ldots, K$ and $\sum_{j=1}^{K} h_{x_{j}}=1$. Since $\mathbf{h}$ and $\boldsymbol{\theta}$ are probability vectors, i.e., their entries are nonnegative and sum up to one, it follows that $\mathrm{D}(\mathbf{h} \| \boldsymbol{\theta}) \triangleq \sum_{i=1}^{K} h_{x_{j}} \log \left(\frac{h_{x_{j}}}{\theta_{x_{j}}}\right) \geq 0$ and $\mathrm{D}(\mathbf{h} \| \boldsymbol{\theta})=0$ if and only if $\boldsymbol{\theta}=\mathbf{h}$ [10]. Therefore, we get that $\sum_{j=1}^{K} h_{x_{j}} \log \theta_{x_{j}}=$ $-\mathrm{D}(\mathbf{h} \| \boldsymbol{\theta})+\sum_{j=1}^{K} h_{x_{j}} \log h_{x_{j}} \leq \sum_{j=1}^{K} h_{x_{j}} \log h_{x_{j}}$, where the equality is reached if and only if $\boldsymbol{\theta}=\mathbf{h}$. Hence, it follows that $\hat{\boldsymbol{\theta}}_{\mathrm{ML}}=$ $\arg \max _{\boldsymbol{\theta}} \sum_{j=1}^{K} h_{x_{j}} \log \theta_{x_{j}}=\mathbf{h}$, i.e., we estimate the probability mass vector $\mathbf{p}_{1}$ with $\mathbf{h}=\left[\frac{N_{1}}{N}, \ldots, \frac{N_{K}}{N}\right]$ at each investment period $N$.

\section{Simulations}

In this section, we illustrate the performance of the proposed algorithm using historical data. We first compare the performance of the proposed algorithm with respect to the conventional methods using the historical data from [8,19], which is collected from the New York Stock Exchange (NYSE) over a 22-year period between 1963-1985. This NYSE data includes 34 stocks (where the Kin Ark stock is excluded) and 5651 investment periods (days). We then use the recent S\&P 500 data to compare the performances of the our algorithm, state-of-the-art algorithms, and the conventional algorithms. In particular, we consider the Consol Energy Inc. (CNX) - Metlife Inc. (MET), Fidelity National Information Services (FIS) - United Parcel Service Inc. (UPS), Fluor Corporation (FLS) - Allegheny Technologies (ATI), Reynolds American Inc. (RAI) - eBay Inc. (EBAY) stock pairs over the period between June 20, 2001 and May 29, 2015 (i.e., 3505 days), and present the average wealth returns. These stocks are chosen since their cumulative returns over the corresponding time interval are between 1-4 dollars per one dollar investment. Finally, we consider the Dow Jones Industrial Average (DJIA) data. We use the alphabetically sorted stock pairs of the DJIA data over the period between July 1 , 2009 and August 7, 2015, and present the average wealth returns. The proposed algorithms are assumed to be able to trade at the following approximated Volume Weighted Average Price (VWAP): $0.4 \times$ Open $+0.6 \times$ Close, where "Open" and "Close" are the opening and closing prices of the stocks, respectively. For all our data sets, adjustments were made for splits and dividends.

Throughout the experiments, the proposed TRP algorithm is constructed as follows. The algorithm introduced in Section 3.1 requires the sample space of the price relative sequences. This sample space is constructed by quantizing the observed realizations into 11 different bins, where each bin is created according to Theorem 1 . We then consider a sliding window of length 100 and 
Accumulated Wealth of the Proposed Algorithms Under 1\% Transaction Cost

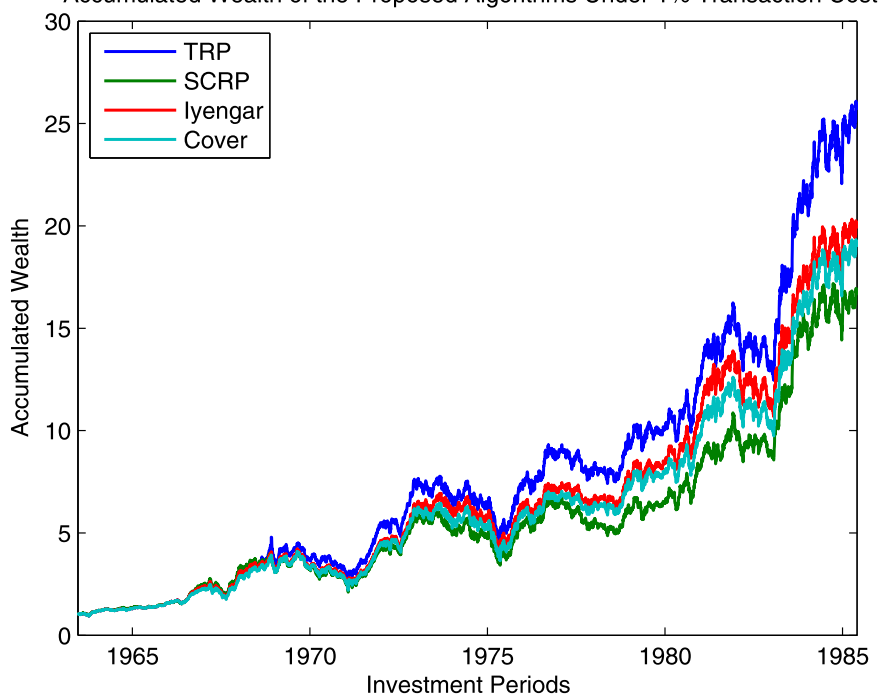

Fig. 2. Accumulated wealths of the proposed algorithms for the NYSE data between 1963-1985 under 1\% transaction cost.

approximate the probability mass vectors of the price relative sequences using the past data for each window. The proposed TRP algorithm constructs its trading rule by maximizing the expected achieved wealth over $b$ and $\epsilon$ according to the discussions in Section 3.5, and we invest using this TRP for each window.

We compare the performance of the proposed TRP algorithm with respect to the state-of-the-art and conventional methods. These competitor algorithms can be briefly described as follows.

- Cover is the Cover's universal portfolio algorithm in [8]. In this method, the wealth is distributed among various stocks according to their wealth returns.

- SCRP is the semiconstant rebalanced portfolio algorithm of [19]. In this method, portfolio is rebalanced only on selected instants to mitigate the effects of transaction costs.

- Iyengar is the universal threshold rebalanced portfolio algorithm of [15]. In this method, the wealth is distributed among various target portfolio and threshold pairs according to their wealth returns.

- CWMR is the confidence weighted mean reversion algorithm of [22]. In this method, the portfolio is modeled as a Gaussian distribution and trading is performed using the mean reversion principle.

- OLMAR is the online portfolio selection with moving average reversion algorithm of [23]. This method is based on a multiple-period mean reversion strategy.

- Anticor is the anti-correlation algorithm of [5]. This method uses the predictable statistical relations between stocks to beat the best stock in the market.

- M0 is the Markov of order zero algorithm of [4]. This method uses the add-beta prediction rule on the Kelly projections of the market vectors.

In Figs. 2 and 3, we present the average wealth returns of the TRP, Cover, SCRP, and Iyengar algorithms over 10 randomly chosen stock pairs from the NYSE data under $1 \%$ and $2 \%$ transaction costs, respectively. These figures illustrate that the proposed TRP algorithm significantly outperforms the competitor algorithms and achieve higher wealth returns. Since the TRP algorithm aims to mitigate the effects of hefty transaction costs, its performances is not significantly affected by a change in the commission rate. The proposed TRP algorithm achieves this robustness by avoiding excessive rebalancings. Although the SCRP and Iyengar algorithms are

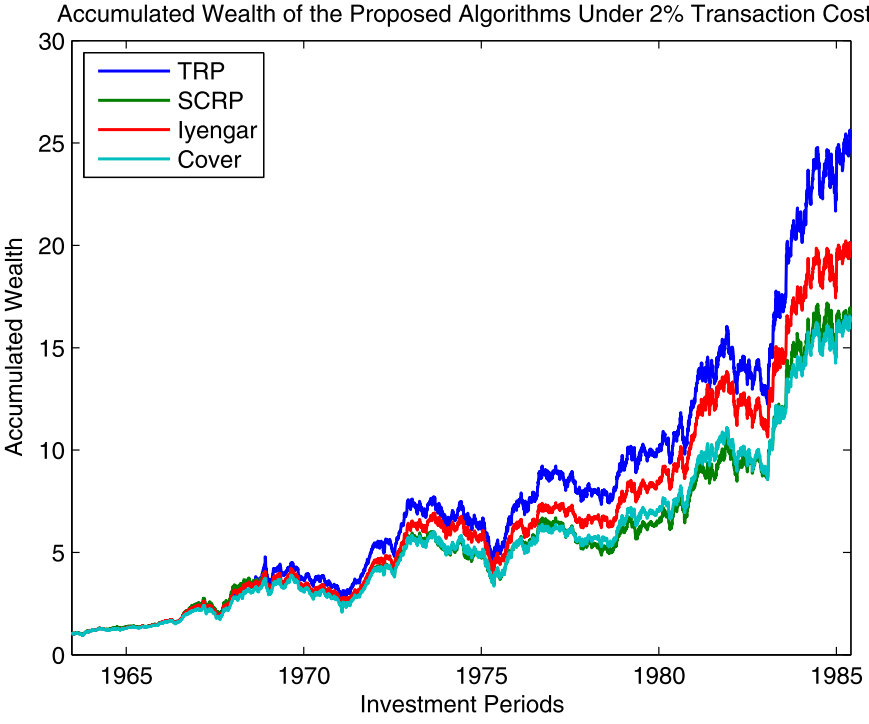

Fig. 3. Accumulated wealths of the proposed algorithms for the NYSE data between 1963-1985 under $2 \%$ transaction cost.

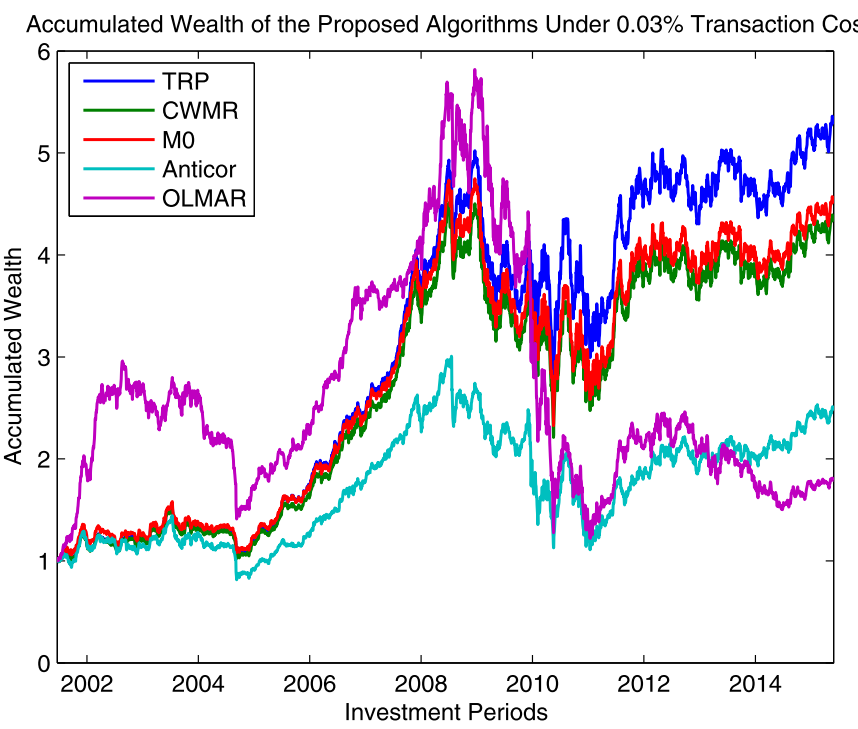

Fig. 4. Accumulated wealths of the proposed algorithms for the S\&P500 data between 2001-2015 under 0.03\% transaction cost.

also robust against transaction costs, the TRP algorithm provides a better performance since it directly aims to maximize the expected achieved wealth.

We next compare the performance of the proposed TRP algorithm with respect to the state-of-the-art algorithms using the recent S\&P 500 data. In Figs. 4 and 5, we have presented the wealth returns of the proposed algorithms for transaction costs of $0.03 \%$ and $0.1 \%$, respectively. As shown in these figures, the proposed TRP algorithm significantly outperforms the state-of-the-art competitor algorithms and achieve the highest final wealth returns. For this experiment, we also provide the Sharpe, Calmar, and Sortino ratios of the proposed algorithms in Table 1, where the values in the table are found according to the model described in $[21,22]$. This table illustrates that the proposed TRP algorithm achieves the highest reward-to-variability ratios. Furthermore, as the transaction cost increases, the Sharpe ratio of our algorithm does not significantly decrease, whereas the Sharpe ratios of the competitor algorithms notably decreases. This illustrates the robustness of the proposed algorithm against transaction costs. Since the proposed algorithm optimizes its trading model with respect 
Table 1

Risk of the proposed algorithms under different transaction costs for the S\&P500 data between 2001-2015.

\begin{tabular}{|c|c|c|c|c|c|c|}
\hline Transaction cost & & TRP & CWMR & M0 & Anticor & OLMAR \\
\hline \multirow[t]{3}{*}{$0.1 \%$} & Sharpe ratio & 0.3382 & 0.2750 & 0.2914 & 0.0116 & -0.0319 \\
\hline & Calmar ratio & 0.2118 & 0.1860 & 0.1899 & 0.0642 & 0.0304 \\
\hline & Sortino ratio & 1.5686 & 1.4149 & 1.4515 & 0.7653 & 0.6979 \\
\hline \multirow[t]{3}{*}{$0.03 \%$} & Sharpe ratio & 0.3404 & 0.2839 & 0.3001 & 0.0669 & 0.0142 \\
\hline & Calmar ratio & 0.2128 & 0.1899 & 0.1937 & 0.0885 & 0.0507 \\
\hline & Sortino ratio & 1.5738 & 1.4347 & 1.4722 & 0.8965 & 0.8095 \\
\hline
\end{tabular}

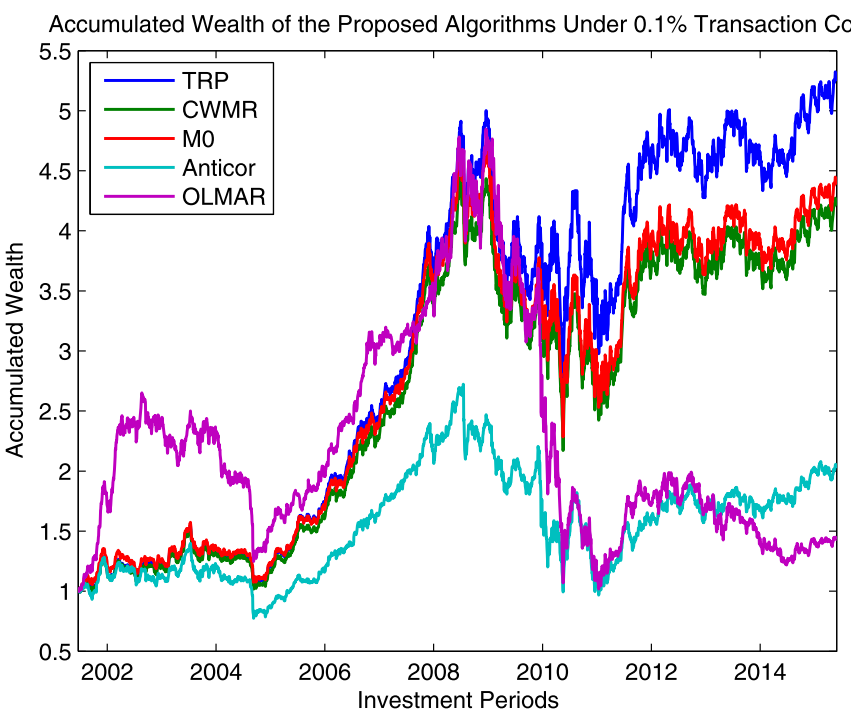

Fig. 5. Accumulated wealths of the proposed algorithms for the S\&P500 data between 2001-2015 under 0.1\% transaction cost.

to a given transaction cost, the risk of the algorithm does not vary excessively.

Although the transaction costs in Figs. 4 and 5 are realistic for institutional trading, the transaction costs for retail trading are around 1-3\%. Since these state-of-the-art algorithms does not optimize their trading model according to the transaction cost, their returns tend to go to zero as the transaction cost exceeds $1 \%$. Thus, we have compared the performance of our algorithm with respect to only the conventional trading algorithms for $3 \%$ transaction cost in Fig. 6. For mild transaction costs (such as the ones in Figs. 4 and 5), the final wealth return of the TRP algorithm is around 5.25 dollars per one dollar investment. On the other hand, for a hefty transaction cost (such as the one in Fig. 6), the total wealth return of the TRP algorithm is around 4.75 dollars per one dollar investment. This illustrates that although the state-of-the-art trading algorithms cannot provide a reasonable wealth return for such hefty transaction costs, the performance of the proposed TRP algorithm is not significantly deteriorated as the transaction cost increases.

We also provide the wealth returns of the proposed TRP algorithm and the conventional algorithms under no transaction cost over the S\&P 500 data for a fair comparison. Fig. 7 illustrates that the algorithms that consider the effects of transaction cost on the wealth return, namely the TRP, SCRP, and lyengar algorithms, provide similar returns for different transaction costs. On the other hand, the performances of the algorithms that do not take transaction cost into account (e.g., the Cover algorithm) are highly volatile with respect to the transaction cost. Although the returns of the SCRP and lyengar algorithms are not significantly affected by the transaction cost, the proposed TRP algorithm outperforms these methods for both no transaction cost and 3\% transaction cost cases.

Finally, in Fig. 8, we present the average wealth returns of the TRP, CWMR, M0, Anticor, and OLMAR algorithms under $1 \%$ trans-

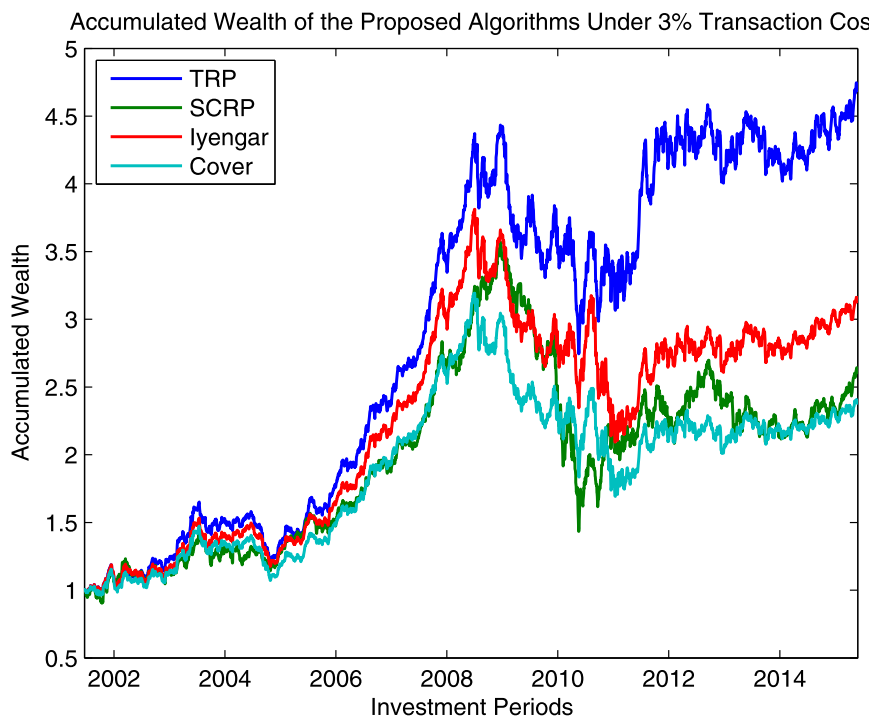

Fig. 6. Accumulated wealths of the proposed algorithms for the S\&P500 data between 2001-2015 under 3\% transaction cost.

Accumulated Wealth of the Proposed Algorithms Under No Transaction Cost

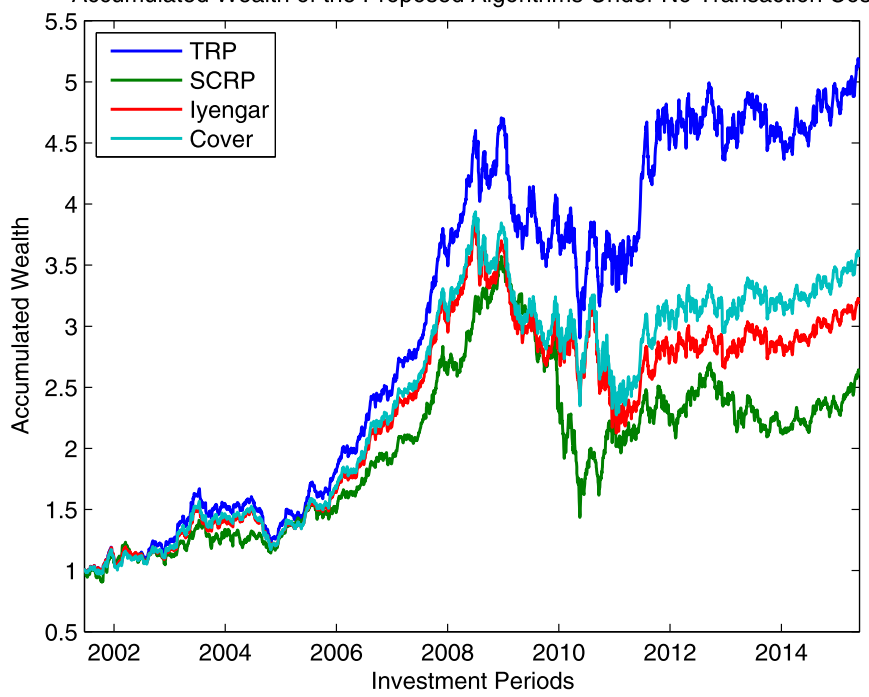

Fig. 7. Accumulated wealths of the proposed algorithms for the S\&P500 data between 2001-2015 under no transaction cost

action cost. As can be seen from this figure, the TRP algorithm provides a superior performance with respect to the competitor algorithms that do not consider the effects of transaction costs. This result highlights the importance of designing portfolio optimization algorithms that avoid excessive number of rebalancings when the transaction cost is high. In Fig. 9, we present the average wealth returns of the TRP, Iyengar, Cover, and SCRP algorithms under $0.5 \%$ transaction cost. Although the performances of the proposed algorithms are comparable with one another since the trans- 


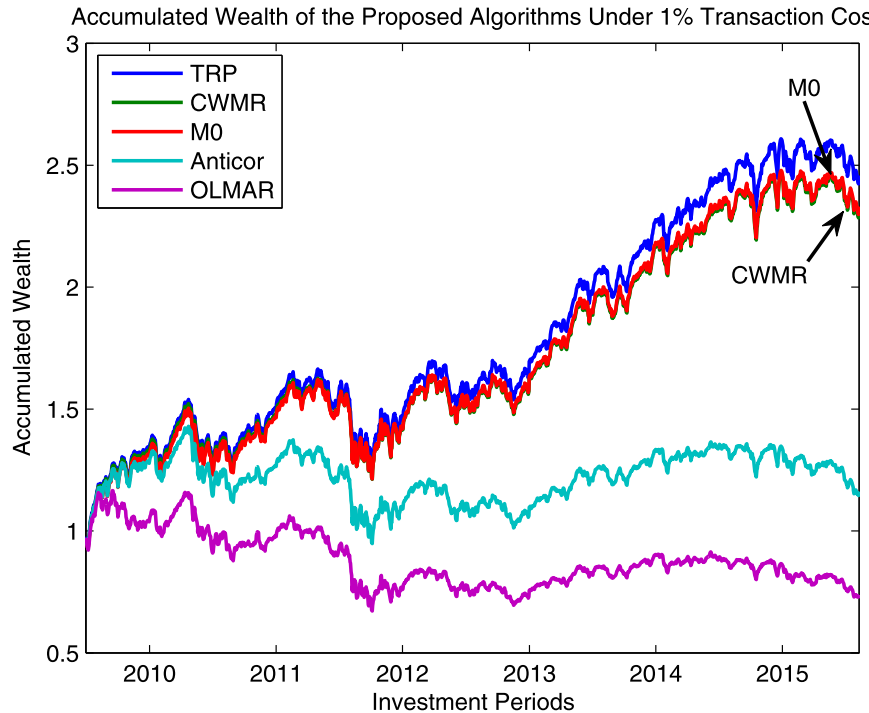

Fig. 8. Accumulated wealths of the proposed algorithms for the DJIA data between 2009-2015 under 1\% transaction cost.

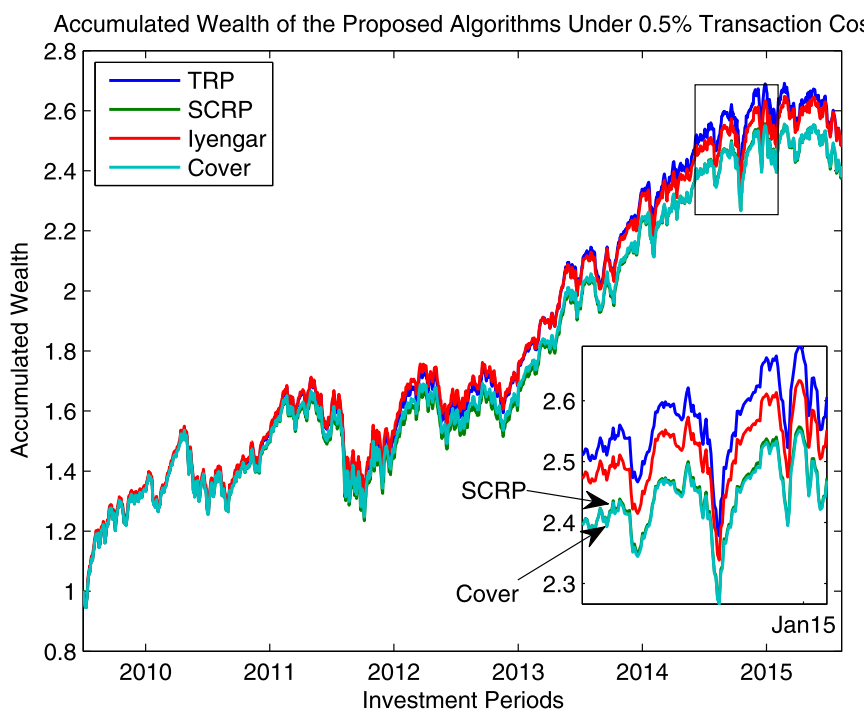

Fig. 9. Accumulated wealths of the proposed algorithms for the DJIA data between 2009-2015 under $0.5 \%$ transaction cost.

action cost is small, the TRP algorithm still provides a marginally higher return with respect to the competitor algorithms.

\section{Conclusion}

We study growth optimal investment in i.i.d. discrete-time markets under proportional transaction costs. Under this market model, we study threshold portfolios that are shown to yield the optimal growth. We first introduce a recursive update to calculate the expected growth for a two-asset market and then extend our results to markets having more than two assets. We next demonstrate that under the threshold rebalancing framework, the achievable set of portfolios form an irreducible Markov chain under mild technical conditions. We evaluate the corresponding stationary distribution of this Markov chain, which provides a natural and efficient method to calculate the cumulative expected wealth. Subsequently, the corresponding parameters are optimized using a brute force approach yielding the growth optimal investment portfolio under proportional transaction costs in i.i.d. discrete-time two-asset markets. We also solve the optimal portfolio selection in discrete-time markets constructed by sampling continuous-time Brownian markets. For the case that the underlying discrete distributions of the price relative vectors are unknown, we provide a maximum likelihood estimator. In our simulations, we use the historical NYSE, S\&P 500, and DJIA data and observe that the introduced TRP algorithm significantly improves the wealth return under both mild and hefty transaction costs.

\section{Appendix A. Proof of Lemma 1}

Consider that an investor invests with a $\operatorname{TRP}(b, \epsilon)$ for $N$ investment periods, where we denote a portfolio sequence realization by $\left\{b(n)=b_{n}\right\}_{n=1}^{N}$. Let us partition the set of $N$-period market scenarios according to the last time the portfolio leaves the interval $(b-\epsilon, b+\epsilon)$. Our claim is that any achievable portfolio at period $N$ can be achieved by an $N$-period market scenario where the portfolio does no leave the interval $(b-\epsilon, b+\epsilon)$ for $N$ periods. To illustrate this, with an abuse of notation, we let $\mathcal{P}$ denote the set of $\mathrm{N}$-period market scenarios, i.e.,

$\mathcal{P} \triangleq\left\{\left\{b_{n}\right\}_{n=1}^{N}: b_{n} \in \mathcal{B}_{n}, n=1, \ldots, N\right\}=\bigcup_{i=1}^{N+1} \mathcal{P}_{i}$,

where $\mathcal{P}_{i}$ is the set of $N$-period market scenarios where the portfolio leaves the interval $(b-\epsilon, b+\epsilon)$ at period $i$ for the last time, $i=1, \ldots, N$, and $\mathcal{P}_{N+1}$ is the set of $N$-period market scenarios where the portfolio does not leave the interval $(b-\epsilon, b+\epsilon)$ for $N$ investment periods. We point out that $\mathcal{P}_{i}$ 's are disjoint, i.e., $\mathcal{P}_{i} \cap \mathcal{P}_{j}=\emptyset$ for $i \neq j$, and their union gives the set of all $N$-period market scenarios, i.e., $\bigcup_{i=1}^{N+1} \mathcal{P}_{i}=\mathcal{P}$.

Let $\mathcal{L}_{i}$ denote the last elements of the sequences in $\mathcal{P}_{i}$, i.e., $\mathcal{L}_{i} \triangleq\left\{b_{N}:\left\{b_{n}\right\}_{n=1}^{N} \in \mathcal{P}_{i}\right\}$. Then, the set of achievable portfolios at investment period $N$, i.e., $\mathcal{B}_{N}$, can be found as follows $\mathcal{B}_{N}=\bigcup_{i=1}^{N+1} \mathcal{L}_{i}$. Hence, our aim is to show that $\mathcal{L}_{i} \subset \mathcal{L}_{N+1}$, $\forall i \in\{1, \ldots, N\}$. To prove this, consider an arbitrary portfolio sequence $\left\{b_{n}\right\}_{n=1}^{N} \in \mathcal{P}_{i}$ for some $i=1, \ldots, N$, thus we have $b_{i}=b$ and $b_{N} \in \mathcal{B}_{N}$, i.e., this sequence results in an achievable portfolio $b_{N} \in \mathcal{L}_{i}$. Now, let us consider another $N$-period market scenario $\left\{b_{n}^{\prime}\right\}_{n=1}^{N} \in \mathcal{P}_{N+1}$, where

$b_{j}^{\prime}= \begin{cases}b, & \text { if } j=1, \ldots, i \\ b_{j}, & \text { if } j=i+1, \ldots, N,\end{cases}$

i.e., we have $X_{1}^{\prime}(n)=X_{2}^{\prime}(n), \forall n \in\{1, \ldots, i\}$. Then, we have $b_{N}^{\prime}=b_{N}$ and consequently $b_{N} \in \mathcal{L}_{N+1}$. Therefore, we conclude that $\mathcal{L}_{i} \subset$ $\mathcal{L}_{N+1}, \forall i \in\{1, \ldots, N\}$.

As $\mathcal{L}_{i} \subset \mathcal{L}_{N+1}, \forall i \in\{1, \ldots, N\}$, we have $\mathcal{B}_{N}=\mathcal{L}_{N+1}$. Then, to determine $\left|\mathcal{B}_{N}\right|$, we consider that the portfolio never leaves the interval $(b-\epsilon, b+\epsilon)$ for $N$ investment periods, where

$b(N)=\frac{b \prod_{i=1}^{N} X_{1}(n)}{b \prod_{i=1}^{N} X_{1}(n)+(1-b) \prod_{i=1}^{N} X_{2}(n)}$.

Let us consider the reciprocal of $b(N)$ and write

$$
\begin{aligned}
\frac{1}{b(N)} & =1+\frac{1-b}{b} \prod_{n=1}^{N} \frac{X_{2}(n)}{X_{1}(n)} \\
& =1+\frac{1-b}{b} e^{\sum_{n=1}^{N} Z(n)},
\end{aligned}
$$

where $Z(n) \triangleq \ln \left(X_{2}(n) / X_{1}(n)\right)$. Then, we observe that the number of different values that the portfolio $b(N)$ can take is the same as the number of different values that the sum $\sum_{n=1}^{N} Z(n)$ can take. Since the price relative sequences $X_{1}(n)$ and $X_{2}(n)$ are elements of the same sample space $\mathcal{X}$ with $|\mathcal{X}|=K$, it follows that $|\mathcal{Z}|=$ 
$M \leq K^{2}-K+1$. Since the number of different values that the $\operatorname{sum} \sum_{n=1}^{N} Z(n)$ can take is equal to $\left(\begin{array}{c}N+M-1 \\ M-1\end{array}\right)$ and $M \leq K^{2}-K+1$, it follows that the number of achievable portfolios at period $N$ is bounded by $\left(\begin{array}{c}N+K^{2}-K \\ K^{2}-K\end{array}\right)$, i.e., $\left|\mathcal{B}_{N}\right|=M_{N} \leq\left(\begin{array}{c}N+K^{2}-K \\ K^{2}-K\end{array}\right)$.

\section{Appendix B. Proof of Lemma 2}

If the portfolio does not leave the interval $(b-\epsilon, b+\epsilon)$ for $N$ investment periods, then

$b(n)=\frac{b \prod_{i=1}^{n} X_{1}(i)}{b \prod_{i=1}^{n} X_{1}(i)+(1-b) \prod_{i=1}^{n} X_{2}(i)} \in(b-\epsilon, b+\epsilon)$,

$\forall n \in\{1, \ldots, N\}$. Taking the reciprocal of $b(n)$, we obtain

$$
\frac{b(1-b-\epsilon)}{(1-b)(b+\epsilon)}<\prod_{i=1}^{n} \frac{X_{2}(i)}{X_{1}(i)}<\frac{b(1-b+\epsilon)}{(1-b)(b-\epsilon)} \text {. }
$$

Noting that $\frac{X_{2}(i)}{X_{1}(i)}=e^{Z(i)}$ and taking the logarithm of each side, it follows that

$\underbrace{\ln \frac{b(1-b-\epsilon)}{(1-b)(b+\epsilon)}}_{:=\alpha^{-}}<\sum_{i=1}^{n} Z(i)<\underbrace{\ln \frac{b(1-b+\epsilon)}{(1-b)(b-\epsilon)}}_{:=\alpha^{+}}$,

i.e., $\sum_{i=1}^{n} Z(i) \in\left(\alpha^{-}, \alpha^{+}\right), \forall n \in\{1, \ldots, N\}$. Hence, if the portfolio leaves the interval $(b-\epsilon, b+\epsilon)$ for the first time at period $k$ for some $k \in\{1, \ldots, N\}$, then we conclude that we have either $b(k) \leq$ $b-\epsilon$ or $b(k) \geq b+\epsilon$; and consequently we have either $\sum_{i=1}^{k} Z(i) \leq$ $\alpha^{-}$or $\sum_{i=1}^{k} Z(i) \geq \alpha^{+}$, i.e., $\sum_{i=1}^{k} Z(i) \notin\left(\alpha^{-}, \alpha^{+}\right)$.

\section{Appendix C. Proof of Lemma 3}

In Lemma 1, we showed that for any investment period $N$, the number of different portfolio values that $b(N)$ can take is equal to the number of different values that the sum $\sum_{n=1}^{N} Z(n)$ can take where $\sum_{n=1}^{k} Z(n) \in\left(\alpha^{-}, \alpha^{+}\right)$for $k=1, \ldots, N$. Since this is true for any investment period $N$, it follows that the number of all achievable portfolios is equal to the number of different values that the sum $\sum_{n=1}^{N} Z(n)$ can take for any $N \in \mathbb{N}$ such that $\sum_{n=1}^{N} Z(n) \in\left(\alpha^{-}, \alpha^{+}\right)$.

Here, we show that if $m \in \mathcal{M} \cap\left(\alpha^{-}, \alpha^{+}\right)$, then there exists a sequence $\left\{Z^{(n)}\right\}_{n=1}^{N} \in \mathcal{Z}$ for some $N \in \mathbb{N}$ such that $m=\sum_{n=1}^{N} Z^{(n)}$ and $\sum_{n=1}^{k} Z^{(n)} \in\left(\alpha^{-}, \alpha^{+}\right)$for $k=1, \ldots, N$.

Let $y \in \mathcal{Y} \cap\left(\alpha^{-}, \alpha^{+}\right)$be an arbitrary element. Then, we have $y=\sum_{i=1}^{M^{+}} y_{i} z_{i}$ for some $y_{i} \in \mathbb{Z}$ and $z_{i} \in \mathcal{Z}^{+}$, where $i=1, \ldots, M^{+}$. We define $S(k) \triangleq \sum_{n=1}^{k} Z^{(n)}$ for $k \geq 1$ and construct a sequence $\left\{Z^{(n)}\right\}_{n=1}^{N} \in \mathcal{Z}$ for some $N \in \mathbb{N}$ such that $y=\sum_{n=1}^{N} Z^{(n)}$ and $S(k) \in$ $\left(\alpha^{-}, \alpha^{+}\right)$for each $k=1, \ldots, N$ as follows. We first let $S(0)=0$, where we obviously have $S(0) \in\left(\alpha^{-}, \alpha^{+}\right)$as $\alpha^{-}<0$ and $\alpha^{+}>0$. Then, for any $k \geq 0$, we iteratively construct our sequence as follows:

- If $S(k) \geq 0$ and

$\circ \exists z_{i} \in \mathcal{Z}^{+}$such that $y_{i}<0$, then we let $Z^{(k+1)}=-z_{i}$ and increase $y_{i}$ by 1 . Note that $S(k+1) \in\left(\alpha^{-}, \alpha^{+}\right)$since $S(k), Z^{(k+1)} \in\left(\alpha^{-}, \alpha^{+}\right), S(k) \geq 0$, and $Z^{(k+1)}<0$.

○ $\forall z_{i} \in \mathcal{Z}^{+}$we have $y_{i} \geq 0$, then we consider set $\mathcal{Z}_{\mathcal{Y}^{+}} \triangleq$ $\left\{z_{i} \in \mathcal{Z}^{+}: y_{i}>0\right\}$. Using the elements of $\mathcal{Z}_{\mathcal{Y}^{+}}$, we construct an arbitrary sequence, in which each $z_{j} \in \mathcal{Z}_{\mathcal{Y}^{+}}$is repeated $y_{j}$ times. We then set $Z^{(k+i)}$ as the $i$ th entry of this sequence. As we know from the previous step that $S(k) \in\left(\alpha^{-}, \alpha^{+}\right)$and we also know that $y \in \mathcal{Y} \cap\left(\alpha^{-}, \alpha^{+}\right)$, i.e., $S(N) \in\left(\alpha^{-}, \alpha^{+}\right)$, then we can conclude that $S(k+i) \in$ $\left(\alpha^{-}, \alpha^{+}\right), \forall k+i \leq N$.
- $S(k)<0$ and

$\circ \exists z_{i} \in \mathcal{Z}^{+}$such that $y_{i}>0$, then we let $Z^{(k+1)}=z_{i}$ and decrease $y_{i}$ by 1 . Note that $S(k+1) \in\left(\alpha^{-}, \alpha^{+}\right)$since $S(k), Z^{(k+1)} \in\left(\alpha^{-}, \alpha^{+}\right), S(k)<0$, and $Z^{(k+1)} \geq 0$.

$\circ \forall z_{i} \in \mathcal{Z}^{+}$we have $y_{i} \leq 0$, then we consider the set $\mathcal{Z}_{\mathcal{Y}^{-}} \triangleq$ $\left\{z_{i} \in \mathcal{Z}^{+}: y_{i}<0\right\}$. Using the elements of $\mathcal{Z}_{\mathcal{Y}^{-}}$, we construct an arbitrary sequence, in which each $z_{j} \in \mathcal{Z}_{\mathcal{Y}^{-}}$is repeated $y_{j}$ times. We then set $Z^{(k+i)}$ as the $i$ th entry of this sequence. As we know from the previous step that $S(k) \in\left(\alpha^{-}, \alpha^{+}\right)$and we also know that $y \in \mathcal{Y} \cap\left(\alpha^{-}, \alpha^{+}\right)$, i.e., $S(N) \in\left(\alpha^{-}, \alpha^{+}\right)$, then we can conclude that $S(k+i) \in$ $\left(\alpha^{-}, \alpha^{+}\right), \forall k+i \leq N$.

Therefore, we can write $y=\sum_{n=1}^{N} Z^{(n)}$, where $N=\sum_{i=1}^{M^{+}} y_{i}$, $\left\{Z^{(n)}\right\}_{n=1}^{N} \in \mathcal{Z}$, and $\sum_{n=1}^{k} Z^{(n)} \in\left(\alpha^{-}, \alpha^{+}\right), \forall k \in\{1, \ldots, N\}$.

\section{Appendix D. Proof of Theorem 1}

We first let $\mathcal{H}$ denote the set of values that the sum $\sum_{n=1}^{N} Z(n) \in\left(\alpha^{-}, \alpha^{+}\right)$can take for any $N \in \mathbb{N}$, i.e., $\mathcal{H} \triangleq\left\{\sum_{n=1}^{N} Z^{(n)}\right.$ : $\left.\left\{Z^{(n)}\right\}_{n=1}^{N} \in \mathcal{Z}, \sum_{n=1}^{k} Z^{(n)} \in\left(\alpha^{-}, \alpha^{+}\right), \forall k\{1, \ldots, N\}, \forall N \in \mathbb{N}\right\}$. Then, assuming that the minimum positive element $\delta$ exists, we next show that $\forall\left\{Z^{(n)}\right\}_{n=1}^{N} \in \mathcal{Z}$, we have $\sum_{n=1}^{N} Z^{(n)}=k \delta$, for some $k \in \mathbb{Z}$.

Assume the contrary that $\exists\left\{Z^{(n)}\right\}_{n=1}^{N} \in \mathcal{Z}$ such that $Z=$ $\sum_{n=1}^{N} Z^{(n)} \neq k \delta$ for any $k \in \mathbb{Z}$. Then, if we divide the real line into intervals of length $\delta$, then $Z$ should lie in one of the intervals, i.e., $\exists k \in \mathbb{Z}$ such that $k \delta<Z<(k+1) \delta$ so that we can write $Z=k \delta+\eta$ where $0<\eta<\delta$. According to the definition of $\mathcal{Y}, \forall y \in \mathcal{Y}$ and $\forall c \in \mathbb{Z}$, we have $c y \in \mathcal{Y}$. Therefore, we can conclude that $k \delta \in \mathcal{Y}$ as $\delta \in \mathcal{Y}$. Furthermore, according to the definition of $\mathcal{Y}, \forall y_{1}, y_{2} \in \mathcal{Y}$, we have $y_{1}-y_{2} \in \mathcal{Y}$. Then, as $Z, k \delta \in \mathcal{Y}$, we have $\eta=Z-k \delta \in \mathcal{Y}$. However, this contradicts the fact that $\delta$ is the minimum positive element of $\mathcal{Y}$ since $0<\eta<\delta$ and $\eta \in \mathcal{Y}$. Therefore, it follows that $\forall h \in \mathcal{H}, \exists k \in \mathbb{Z}$ such that $h=k \delta$. As we also have $\forall h \in \mathcal{H}$, $h \in\left(\alpha^{-}, \alpha^{+}\right)$, then it follows that there are finitely many elements in $\mathcal{H}$. As $|\mathcal{B}|=|\mathcal{H}|$ according to Lemma 1 , it follows that the set of achievable portfolios $\mathcal{B}$ is also finite.

If a minimum element $\delta$ does not exist, our claim is that $\mathcal{B}$ contains countably infinitely many elements. Since every finite set of real numbers has a minimum, there are either infinitely many positive elements in the set $\mathcal{Y}$ or none. We know that there exists $z_{i} \neq 0$ so that there are positive numbers in $\mathcal{Y}$. Therefore, there are infinitely many elements in $\mathcal{Y}$. Now assume that $\exists \gamma_{1}>0$ such that $\gamma_{1}=\sum_{n=1}^{N} Z^{(n)}$ for some $N \in \mathbb{N}$, where $\left\{Z^{(n)}\right\}_{n=1}^{N} \in \mathcal{Z}$ and $\sum_{n=1}^{k} Z^{(n)} \in\left(\alpha^{-}, \alpha^{+}\right)$. Then, by Lemma 3 , it follows that $\gamma_{1} \in \mathcal{Y} \cap\left(0, \alpha^{+}\right)$and since there exists no positive minimum element of $\mathcal{Y}, \exists \gamma_{2}>0$ such that $\gamma_{2}<\gamma_{1}$ and $\gamma_{2} \in \mathcal{Y} \cap\left(0, \alpha^{+}\right)$. Continuing in this manner, we can construct a decreasing sequence $\left\{\gamma_{n}\right\}$ such that $\gamma_{n} \in \mathcal{Y} \cap\left(0, \alpha^{+}\right), \forall n \in \mathbb{N}$. Note that we have $\gamma_{n} \in \mathcal{H}$, $\forall n \in \mathbb{N}$ from Lemma 3 , so that there are countably infinite elements in $\mathcal{H}$. Hence, it follows that $\mathcal{B}$ has countably infinitely many elements.

\section{Appendix E. Proof of Corollary 1}

Consider an arbitrary sequence $\left\{Z^{(n)}\right\}_{n=1}^{N} \in \mathcal{Z}$ for some $N \in \mathbb{N}$ such that $Z=\sum_{n=1}^{N} Z^{(n)}>0$ and $\sum_{n=1}^{k} Z^{(n)} \in\left(\alpha^{-}, \alpha^{+}\right), \forall k \in$ $\{1, \ldots, N\}$. Then, by Lemma 3 , it follows that $Z \in \mathcal{Y} \cap\left(0, \alpha^{+}\right)$. Since $\delta$ is the minimum positive element of $\mathcal{Y}$, it follows that $0<\delta \leq M$ and $\delta \in \mathcal{Y} \cap\left(0, \alpha^{+}\right)$. Hence, by Lemma 3 , we conclude that $\delta=\sum_{n=1}^{N^{\prime}} Z^{(n)}$ for some $N^{\prime} \in \mathbb{N}$ and $\left\{Z^{(n)}\right\}_{n=1}^{N^{\prime}} \in \mathcal{Z}$, where $\sum_{n=1}^{k} Z^{(n)} \in\left(\alpha^{-}, \alpha^{+}\right), \forall k \in\left\{1, \ldots, N^{\prime}\right\}$. As we showed in Theorem 1 that $\forall y \in \mathcal{Y}$, we have $y=k \delta$ for some $k \in \mathbb{Z}$, then the 
number of elements in $\mathcal{Y} \cap\left(\alpha^{-}, \alpha^{+}\right)$is $\left\lfloor\frac{\alpha^{+}-\alpha^{-}}{\delta}\right\rfloor$. Hence, it follows that there are exactly $\left\lfloor\frac{\alpha^{+}-\alpha^{-}}{\delta}\right\rfloor$ achievable portfolios since Lemma 3 implies that the set $\mathcal{Y}^{\delta} \cap\left(\alpha^{-}, \alpha^{+}\right)$is equivalent to the set of different values that the sum $\sum_{n=1}^{N} Z(n)$ can take $\forall N \in \mathbb{N}$, where $Z(n) \in \mathcal{Z}$ for $\forall n \in\{1, \ldots, N\}$ and $\sum_{n=1}^{k} Z(n) \in\left(\alpha^{-}, \alpha^{+}\right)$, $\forall k \in\{1, \ldots, N\}$, and the cardinality of the latter set is equal to the number of achievable portfolios.

\section{References}

[1] A.J. Bean, A.C. Singer, Factor graphs for universal portfolios, in: 2009 Conference Record of the Forty-Third Asilomar Conference on Signals, Systems and Computers, 2009, pp. 1375-1379.

[2] A.J. Bean, A.C. Singer, Factor graph switching portfolios under transaction costs, in: IEEE International Conference on Acoustics Speech and Signal Processing, 2011, pp. 5748-5751.

[3] A. Blum, A. Kalai, Universal portfolios with and without transaction costs, Mach. Learn. (1997) 309-313.

[4] A. Borodin, R. El-Yaniv, V. Gogan, On the competitive theory and practice of portfolio selection (extended abstract), in: Proceedings of the 4th Latin American Symposium on Theoretical Informatics, LATIN'00, 2000, pp. 173-196.

[5] A. Borodin, R. El-Yaniv, V. Gogan, Can we learn to beat the best stock, J. Artif. Intell. Res. 21 (2004) 579-594.

[6] A. Borodin, R. El-Yaniv, V. Govan, Can we learn to beat the best stock, J. Artif. Intell. Res. 21 (2004) 579-594.

[7] M.W. Brandt, P. Santa-Clara, R.I. Valkanov, Parametric portfolio policies: exploiting characteristics in the cross section of equity returns, in: EFA 2005 Moscow Meetings Paper, 2005

[8] T. Cover, Universal portfolios, Math. Finance 1 (1) (1991) 1-29.

[9] T. Cover, E. Ordentlich, Universal portfolios with side-information, IEEE Trans. Inf. Theory 42 (2) (1996) 348-363.

[10] T. Cover, J.A. Thomas, Elements of Information Theory, Wiley Series, 1991.

[11] M.H.A. Davis, A.R. Norman, Portfolio selection with transaction costs, Math. Oper. Res. 15 (4) (1990) 676-713.

[12] K. Drakakis, Application of signal processing to the analysis of financial data [in the spotlight], IEEE Signal Process. Mag. 26 (5) (September 2009) 158-160.

[13] D.P. Helmbold, R.E. Schapire, Y. Singer, M.K. Warmuth, On-line portfolio selection using multiplicative updates, Math. Finance 8 (1998) 325-347.

[14] G.N. Iyengar, Discrete time growth optimal investment with costs, http://www.ieor.columbia.edu/ gi10/Papers/stochastic.pdf, July 2002.

[15] G.N. Iyengar, Universal investment in market with transaction costs, Math. Finance 15 (2) (2005) 359-371.

[16] A. Kalai, S. Vempala, Efficient algorithms for universal portfolios, in: IEEE Symposium on Foundations of Computer Science, 2000, pp. 486-491.

[17] S.S. Kozat, A.C. Singer, Universal switching portfolios under transaction costs, in: IEEE International Conference on Acoustics, Speech and Signal Processing, 2008, pp. 5404-5407.

[18] S.S. Kozat, A.C. Singer, Switching strategies for sequential decision problems with multiplicative loss with application to portfolios, IEEE Trans. Signal Process. 57 (2009) 2192-2208

[19] S.S. Kozat, A.C. Singer, Universal semiconstant rebalanced portfolios, Math. Finance 21 (2) (2011) 293-311.

[20] S.S. Kozat, A.C. Singer, A.J. Bean, A tree-weighting approach to sequential decision problems with multiplicative loss, Signal Process. 92 (4) (2011) 890-905.

[21] B. Li, S.C.H. Hoi, Online portfolio selection: a survey, ACM Comput. Surv. 46 (3) (January 2014) 35:1-35:36.

[22] B. Li, S.C.H. Hoi, P. Zhao, V. Gopalkrishnan, Confidence weighted mean reversion strategy for online portfolio selection, ACM Trans. Knowl. Discov. Data 7 (1) (March 2013) 4:1-4:38

[23] B. Li, S.C.H. Hoi, D. Sahoo, Z.-Y. Liu, Moving average reversion strategy for online portfolio selection, Artif. Intell. 222 (2015) 104-123.

[24] D. Luenberger, Investment Science, Oxford University Press, 1998.

[25] H. Markowitz, Portfolio selection, J. Finance 7 (1) (March 1952) 77-91.

[26] H. Markowitz, Foundations of portfolio theory, J. Finance 46 (2) (June 1991) 469-477.

[27] C.D. Meyer, Matrix Analysis and Applied Linear Algebra, SIAM, 2001.

[28] H. Niu, J. Wang, Volatility clustering and long memory of financial time series and financial price model, Digit. Signal Process. 23 (2) (2013) 489-498.

[29] A. Ozkaya, A model of active trading by using the properties of chaos, Digit. Signal Process. 39 (2015) 15-21.

[30] I. Pollak, Incorporating financial applications in signal processing curricula, IEEE Signal Process. Mag. 28 (5) (Sept 2011) 122-125.

[31] H. Stark, J.W. Woods, Probability and Random Processes with Applications to Signal Processing, Prentice-Hall, 2001.
[32] M.I. Taksar, M.J. Klass, D. Assaf, Diffusion model for optimal portfolio selection in the presence of brokerage fees, Math. Oper. Res. 13 (2) (1988) 277-294

[33] M.U. Torun, A.N. Akansu, M. Avellaneda, Portfolio risk in multiple frequencies, IEEE Signal Process. Mag. 28 (5) (September 2011) 61-71.

[34] V. Vovk, C. Watkins, Universal portfolio selection, in: Proceedings of the COLT, 1998, pp. 12-23.

[35] W.J. Yoon, K.S. Park, A study on the market instability index and risk warning levels in early warning system for economic crisis, Digit. Signal Process. 29 (2014) 35-44.

N. Denizcan Vanli received the B.S. degree with high honors in electrical and electronics engineering from Bilkent University, Turkey, in 2013.

He is currently working toward the M.S. degree in the Department of Electrical and Electronics Engineering at Bilkent University. His research interests include machine learning, convex optimization, and statistical signal processing.

Sait Tunc received the B.S. degree in electrical and electronics engineering from Bogazici University, Turkey, in 2010 and the M.S. degree in electrical and electronics engineering from Koc University, Turkey, in 2012

He is currently pursuing the Ph.D. degree in industrial and systems engineering at the University of Wisconsin, Madison. His research interests include signal processing, information theory, and Markov decision processes.

Mehmet A. Donmez received the B.S. degrees from both the Department of Electrical and Electronics Engineering and the Department of Mathematics, Bogazici University, Turkey, with honors and M.S. degree from the Department of Electrical and Electronics Engineering, Koc University, Turkey.

He is currently a Ph.D. candidate at the Electrical and Computer Engineering Department, University of Illinois at Urbana-Champaign, under the supervision of Prof. Andrew C. Singer. He is working as part of the Systems on Nanoscale Information fabriCs (SONIC) Center, which is a multi-university research center focusing on the design of robust, energy efficient, and intelligent computing platforms using emerging nanoscale devices that are inspired by the information processing principles found in communication and biological systems. His current research interests include adaptive signal processing, intelligent systems, online machine learning, signal processing for communications, and machine learning for signal processing. He has served as a Reviewer for the IEEE Transactions on Signal Processing and Digital Signal Processing.

Suleyman Serdar Kozat received the B.S. degree with full scholarship and high honors from Bilkent University, Turkey. He received the M.S. and Ph.D. degrees in electrical and computer engineering from University of Illinois at Urbana Champaign, Urbana, IL. Dr. Kozat is a graduate of Ankara Fen Lisesi.

After graduation, Dr. Kozat joined IBM Research, T.J. Watson Research Lab, Yorktown, New York, US as a Research Staff Member in the Pervasive Speech Technologies Group. While doing his Ph.D., he was also working as a Research Associate at Microsoft Research, Redmond, Washington, US in the Cryptography and Anti-Piracy Group. He holds several patent inventions due to his research accomplishments at IBM Research and Microsoft Research. After serving as an Assistant Professor at Koc University, Dr. Kozat is currently an Assistant Professor (with the Associate Professor degree) at the electrical and electronics department of Bilkent University.

Dr. Kozat is a Senior Member of the IEEE and the President of the IEEE Signal Processing Society, Turkey Chapter. He has been elected to the IEEE Signal Processing Theory and Methods Technical Committee and IEEE Machine Learning for Signal Processing Technical Committee, 2013. He has been awarded IBM Faculty Award by IBM Research in 2011, Outstanding Faculty Award by Koc University in 2011 (granted the first time in 16 years), Outstanding Young Researcher Award by the Turkish National Academy of Sciences in 2010, ODTU Prof. Dr. Mustafa N. Parlar Research Encouragement Award in 2011, Outstanding Faculty Award by Bilim Kahramanlari, 2013 and holds Career Award by the Scientific Research Council of Turkey, 2009. 